\title{
How Substrate Solvation Contributes to the Enantioselectivity of Subtilisin Toward Secondary Alcohols
}

Christopher K. Savile ${ }^{\mathrm{a}, \mathrm{b}}$ and Romas J. Kazlauskas*,b

${ }^{a}$ Department of Chemistry, McGill University, 801 Sherbrooke Street West, Montréal, Québec, Canada, H3A 2 K6

${ }^{b}$ Department of Biochemistry, Molecular Biology \& Biophysics and The Biotechnology Institute, University of Minnesota, 1479 Gortner Avenue, St. Paul, MN 55108 USA

*Correspondence to: R. J. Kazlauskas, Department of Biochemistry, Molecular Biology \& Biophysics and the Biotechnology Institute, University of Minnesota, 1479 Gortner Avenue, St. Paul, MN 55108 USA. Tel (612) 624-5904; Fax (612)625-5780 Email: rjk@umn.edu

\section{Supporting Information}

\section{Table of Contents}

General methods

Synthesis of substrates

Determination of absolute configuration

Fermentation and purification of subtilisin $\mathrm{BPN}^{\prime}$ and subtilisin $\mathrm{E}$

Molecular modeling

Tables of enantioselectivity page $\mathrm{S} 1$

page $\mathrm{S} 1$

page $\mathrm{S} 7$

page $S 9$

page $\mathrm{S} 11$

page S14

General Methods. ${ }^{1} \mathrm{H}$ - and ${ }^{13} \mathrm{C}$-NMR spectra were obtained as $\mathrm{CDCl}_{3}$ solutions at 300 $\mathrm{MHz}$ and $75 \mathrm{MHz}$, respectively. Chemical shifts are expressed in ppm ( $\delta$ ) and are referenced to tetramethylsilane or solvent signal. Coupling constants are reported in Hertz (Hz). GC analyses were performed on a $25 \mathrm{~m}$ x $0.25 \mathrm{~mm}$ Chrompack CP-Chiralsil-Dex CB column (Varian Inc., Palo Alto, USA) with $\mathrm{He}$ as carrier gas using one of the three following temperature programs: $\mathbf{A}$ $17.5 \mathrm{psi}, 50^{\circ} \mathrm{C}, 5{ }^{\circ} \mathrm{C} \mathrm{min}{ }^{-1}, 150{ }^{\circ} \mathrm{C}$ held for $5 \mathrm{~min}, 2.5^{\circ} \mathrm{C} \mathrm{min}^{-1} ; 175^{\circ} \mathrm{C}$ held for $5 \mathrm{~min}, 5{ }^{\circ} \mathrm{C} \mathrm{min}^{-}$ ${ }^{1}, 200{ }^{\circ} \mathrm{C}$ held for $30 \mathrm{~min}$ ); B $17.5 \mathrm{psi}, 50{ }^{\circ} \mathrm{C}, 5^{\circ} \mathrm{C} \mathrm{min}^{-1} ; 150{ }^{\circ} \mathrm{C}$ held for $10 \mathrm{~min}, 1.0{ }^{\circ} \mathrm{C} \mathrm{min}{ }^{-1}$,

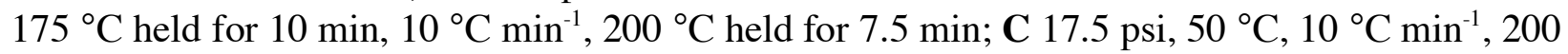
${ }^{\circ} \mathrm{C}$ held for $15 \mathrm{~min}$ ). HPLC analyses were performed on a 4.6 x $250 \mathrm{~mm}$ Daicel Chiralcel OD or Chrialpak AD-H column (Chiral Technologies, Exton, USA) and monitored at $254 \mathrm{~nm}$. Flash chromatography with silica gel (35-75 mesh) was used to purify all intermediates and substrates. All reagents, buffers, starting materials and anhydrous solvents were purchased from SigmaAldrich (Milwaukee, USA) and used without purification. All air- and moisture-sensitive reactions were performed under Ar.

Lipase from Burkholderia cepacia (PCL) ${ }^{1}$ was purchased from Amano Enzyme USA (Troy, USA). The pBE3 Escherichia coli-Bacillus subtilis shuttle vector ${ }^{2}$ containing the subtilisin E gene was kindly provided by Dr. F. Arnold (Caltech, USA) and Bacillus subtilis strain DB104 ${ }^{3}$ was a gift from Dr. S. L. Wong (University of Calgary, Canada).

Synthesis of Substrates. We synthesized esters 1a-13a, 10c, 11b and $12 b$ by treating the corresponding secondary alcohol with the appropriate acid chloride in the presence of pyridine (Scheme S1a). $m$-CPBA oxidation of 1-(pyrid-4-yl) ethanol and 2a gave the 4-pyridine $N$-oxide derivatives $\mathbf{5}$ and $\mathbf{5 a}$, respectively (Scheme $\mathrm{S} 1 \mathrm{~b}$ ). We synthesized secondary alcohols by $\mathrm{NaBH}_{4}$ 
(4 and 6-10) or $\mathrm{LiAlH}_{4}(\mathbf{1 2})$ reduction of the corresponding ketone (Scheme S1c and S1d). The details for ester synthesis are presented first, followed by the secondary alcohols.

(a)<smiles>[R]CC(=O)Cl</smiles>
$\mathrm{R}_{\mathrm{O}} \stackrel{\mathrm{O}}{\mathrm{N}} \mathrm{R}^{1}$ a $\mathrm{R}^{1}=\mathrm{Bn}$ b $\mathrm{R}^{1}=\mathrm{Cl}$ c $\mathrm{R}^{1}=\mathrm{H}$

(b)

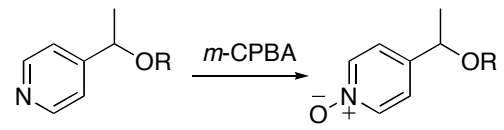

(c)

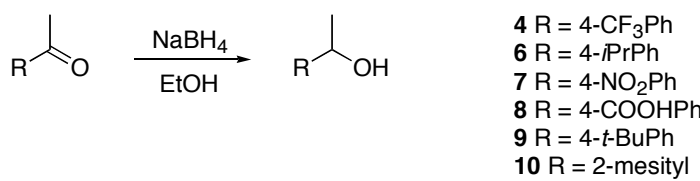

(d)

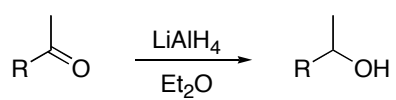

$5 \mathrm{R}=\mathrm{H}$
$5 \mathrm{a} \mathrm{R}=$

$12 \mathrm{R}=2,4,6-\mathrm{t}-i-\mathrm{PrPh}$
$1 \mathrm{a} \mathrm{R}=\mathrm{Ph}$

2a $\mathrm{R}=4$-pyridyl $\quad 9 \mathrm{a} R=4-t-\mathrm{BuPh}$

3a $\mathrm{R}=p$-tolyl $\quad 10 \mathrm{a} \mathrm{R}=1$-naphthyl

4a $\mathrm{R}=4-\mathrm{CF}_{3} \mathrm{Ph} \quad$ 10c $\mathrm{R}=1$-naphthyl

5a $\mathrm{R}=4$-pyridine $\mathrm{N}$-oxide $11 \mathrm{a} \mathrm{R}=2$-mesityl

6a $\mathrm{R}=4-i-\mathrm{PrPh} \quad 11 \mathrm{~b} \mathrm{R}=2$-mesityl

7a $\mathrm{R}=4-\mathrm{NO}_{2} \mathrm{Ph} \quad 12 \mathrm{~b} \mathrm{R}=2,4,6-\mathrm{t}-i-\mathrm{PrPh}$

13a $\mathrm{R}=t-\mathrm{Bu}$

Scheme S1. Synthesis of esters 1a-13a, 10c, 11b and 12b and secondary alcohols 4-10 and 12.

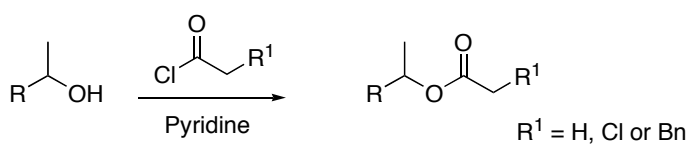

Synthesis of racemic esters 1a-13a. General Procedure. Dihydrocinnamoyl chloride $(15 \mathrm{mmol})$ was added dropwise to a stirred solution of secondary alcohol $(10 \mathrm{mmol})$ and pyridine $(15 \mathrm{mmol})$ in $\mathrm{CH}_{2} \mathrm{Cl}_{2}$ $(50 \mathrm{~mL})$ at $0{ }^{\circ} \mathrm{C}$. The ice bath was removed and stirred until the reaction was complete by TLC. The reaction was quenched with the addition of sat. $\mathrm{NaHCO}_{3}(25 \mathrm{~mL})$ (except 8a). The layers were separated and the aqueous layer was extracted with $\mathrm{CH}_{2} \mathrm{Cl}_{2}(2 \times 25 \mathrm{~mL})$. The combined organic layers were washed with $1 \mathrm{~N} \mathrm{HCl}(2 \times 25 \mathrm{~mL})$ (except $\mathbf{2 a})$, sat. $\mathrm{NaHCO}_{3}(2 \times 25 \mathrm{~mL})$ (except 8a), sat. $\mathrm{NaCl}(25 \mathrm{~mL})$ and dried over $\mathrm{Na}_{2} \mathrm{SO}_{4}$. The organic layer was concentrated in vacuo to give the crude ester. All substrates were purified on silica gel. The relevant analytical data are given below:

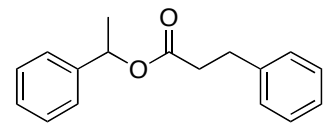

Dihydrocinnamic acid 1-phenyl-ethyl ester (1a). ${ }^{4}$ Purification on silica gel (95:5 hexanes/EtOAc) to give a clear liquid $(4.73 \mathrm{~g}, 75 \%):{ }^{1} \mathrm{H}$ NMR $\delta 1.52\left(\mathrm{~d}, J=6.6,3 \mathrm{H}, \mathrm{CH}_{3}\right), 2.68\left(\mathrm{~m}, 2 \mathrm{H}, \mathrm{C}(\mathrm{O}) \mathrm{CH}_{2}\right), 2.97(\mathrm{t}, J=7.8$, $\left.2 \mathrm{H}, \mathrm{CH}_{2} \mathrm{Ph}\right), 5.90(\mathrm{q}, \mathrm{J}=6.6,1 \mathrm{H}, \mathrm{CH}), 7.19-7.38\left(\mathrm{~m}, 10 \mathrm{H}\right.$, phenyl); ${ }^{13} \mathrm{C}$ NMR $\delta 22.2\left(\mathrm{CH}_{3}\right), 31.0$ $\left(\mathrm{CH}_{2} \mathrm{Ph}\right), 36.2\left(\mathrm{C}(\mathrm{O}) \mathrm{CH}_{2}\right), 72.4(\mathrm{CH}), 126.1,126.3,127.9,128.4,128.5,140.5,141.7$ (phenyl), 172.2 $(C=\mathrm{O})$; HRMS calcd for $\mathrm{C}_{17} \mathrm{H}_{18} \mathrm{O}_{2} \mathrm{Na}[\mathrm{M}+\mathrm{Na}]^{+}$277.1209. Found: 277.1202. The enantiomers were separated using GC (program $\left.\mathbf{A} ;(S)-\mathbf{1 a}, t_{\mathrm{R}}=40.8 \mathrm{~min} ;(R)-\mathbf{1 a}, t_{\mathrm{R}}=41.0 \mathrm{~min}\right)$.

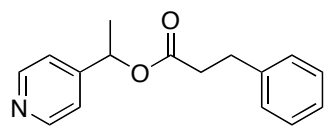

Dihydrocinnamic acid 1-pyridin-4-yl-ethyl ester (2a).

Purification on silica gel (3:1 to $2: 1$ hexanes/EtOAc) to give a viscous, yellow liquid $(3.27 \mathrm{~g}, 85 \%):{ }^{1} \mathrm{H}$ NMR $\delta 1.49\left(\mathrm{~d}, J=6.6,3 \mathrm{H}, \mathrm{CH}_{3}\right), 2.73(\mathrm{~m}$, $\left.2 \mathrm{H}, \mathrm{C}(\mathrm{O}) \mathrm{CH}_{2}\right), 2.99$ (t, $\left.J=7.5,2 \mathrm{H}, \mathrm{CH}_{2} \mathrm{Ph}\right), 5.82(\mathrm{q}, J=6.9,1 \mathrm{H}, \mathrm{CH}), 7.14(\mathrm{~d}, J=6.0,2 \mathrm{H}$, pyridyl), 7.19-7.32 (m, 7H, phenyl), $8.56\left(\mathrm{~d}, J=6.3,2 \mathrm{H}\right.$, pyridyl); ${ }^{13} \mathrm{C}$ NMR $\delta 21.9\left(\mathrm{CH}_{3}\right), 30.9$ $\left(\mathrm{CH}_{2} \mathrm{Ph}\right), 35.9\left(\mathrm{C}(\mathrm{O}) \mathrm{CH}_{2}\right), 70.8(\mathrm{CH}), 120.6,126.4,128.3,128.5,140.2,149.9$, 150.4 (aromatic), 
172.2 $(C=\mathrm{O})$; HRMS calcd for $\mathrm{C}_{16} \mathrm{H}_{18} \mathrm{NO}_{2}[\mathrm{M}+\mathrm{H}]^{+}$256.1343. Found: 256.1342. The enantiomers were separated using GC (program A; $(S)-2 \mathbf{a}, t_{\mathrm{R}}=45.0 \mathrm{~min} ;(R)-\mathbf{2} \mathbf{a}, t_{\mathrm{R}}=45.2 \mathrm{~min}$ ).

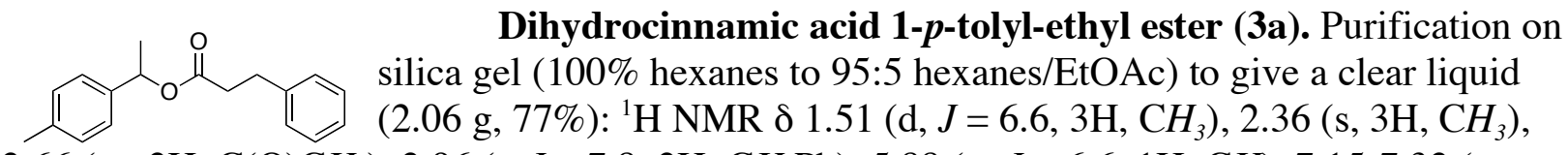
$2.66\left(\mathrm{~m}, 2 \mathrm{H}, \mathrm{C}(\mathrm{O}) \mathrm{CH}_{2}\right), 2.96\left(\mathrm{t}, J=7.8,2 \mathrm{H}, \mathrm{CH}_{2} \mathrm{Ph}\right), 5.88(\mathrm{q}, J=6.6,1 \mathrm{H}, \mathrm{CH}), 7.15-7.32(\mathrm{~m}$, 9H, phenyl); ${ }^{13} \mathrm{C}$ NMR $\delta 21.2\left(\mathrm{CH}_{3}\right), 22.1\left(\mathrm{CH}_{3}\right), 31.0\left(\mathrm{CH}_{2} \mathrm{Ph}\right), 36.2\left(\mathrm{C}(\mathrm{O}) \mathrm{CH}_{2}\right), 72.3(\mathrm{CH})$, 126.1, 126.2 , 128.4, 128.5, 129.2, 137.6, 138.7, 140.6 (phenyl), 172.2 ( $C=\mathrm{O}$ ); HRMS calcd for $\mathrm{C}_{18} \mathrm{H}_{20} \mathrm{O}_{2} \mathrm{Na}[\mathrm{M}+\mathrm{Na}]^{+}$291.1366. Found: 291.1360. The enantiomers were separated using GC (program A; $(S)-3 \mathbf{a}, t_{\mathrm{R}}=44.5 \mathrm{~min} ;(R)-3 \mathbf{a}, t_{\mathrm{R}}=44.6 \mathrm{~min}$ ).

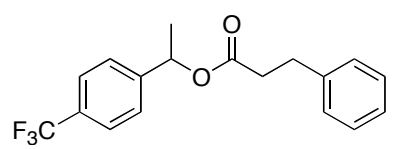

Dihydrocinnamic acid 1-(4-trifluoromethylphenyl)-ethyl ester (4a). Purification on silica gel (100\% hexanes to 95:5 hexanes/EtOAc) to give a clear liquid $(960 \mathrm{mg}, 76 \%):{ }^{1} \mathrm{H}$ NMR $\delta 1.51$ $\left(\mathrm{d}, J=6.6,3 \mathrm{H}, \mathrm{CH}_{3}\right), 2.70\left(\mathrm{~m}, 2 \mathrm{H}, \mathrm{C}(\mathrm{O}) \mathrm{CH}_{2}\right), 2.97(\mathrm{t}, J=7.5,2 \mathrm{H}$, $\left.\mathrm{CH}_{2} \mathrm{Ph}\right), 5.90(\mathrm{q}, \mathrm{J}=6.6,1 \mathrm{H}, \mathrm{CH}), 7.18-7.29\left(\mathrm{~m}, 5 \mathrm{H}\right.$, phenyl), 7.37-7.61 (m, 4H, phenyl); ${ }^{13} \mathrm{C}$ NMR $\delta 22.3\left(\mathrm{CH}_{3}\right), 31.0\left(\mathrm{CH}_{2} \mathrm{Ph}\right), 36.1\left(\mathrm{C}(\mathrm{O}) \mathrm{CH}_{2}\right), 71.7(\mathrm{CH}), 125.6\left(\mathrm{q}, C \mathrm{~F}_{3}\right), 126.3,126.4$, 128.4, 128.6, 140.3, 145.7, (phenyl), $172.1(C=\mathrm{O})$; HRMS calcd for $\mathrm{C}_{18} \mathrm{H}_{18} \mathrm{~F}_{3} \mathrm{O}_{2}[\mathrm{M}+\mathrm{H}]^{+}$ 323.1258. Found: 323.1268. The enantiomers were separated using GC (program $\mathbf{C} ;(S)-4 a, t_{\mathrm{R}}=$ $\left.17.2 \min ;(R)-4 \mathbf{a}, t_{\mathrm{R}}=17.3 \mathrm{~min}\right)$.

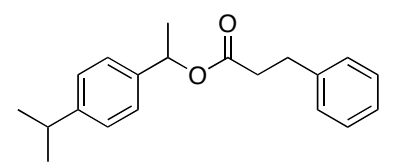

Dihydrocinnamic acid 1-(4-isopropylphenyl)-ethyl ester (6a). Purification on silica gel (100\% hexanes to $95: 5$ hexanes/EtOAc) to give a clear liquid $(1.24 \mathrm{~g}, 84 \%):{ }^{1} \mathrm{H}$ NMR $\delta 1.26(\mathrm{~d}, J=6.9,6 \mathrm{H}$, $\left.\mathrm{CH}\left(\mathrm{CH}_{3}\right)_{2}\right), 1.51\left(\mathrm{~d}, \mathrm{~J}=6.6,3 \mathrm{H}, \mathrm{CH}_{3}\right), 2.66\left(\mathrm{~m}, 2 \mathrm{H}, \mathrm{C}(\mathrm{O}) \mathrm{CH}_{2}\right), 2.93$ (hept, $\left.J=6.6,1 \mathrm{H}, \mathrm{CH}\left(\mathrm{CH}_{3}\right)_{2}\right), 2.97\left(\mathrm{t}, J=7.8,2 \mathrm{H}, \mathrm{CH}_{2} \mathrm{Ph}\right), 5.89$ (q, $\left.J=6.6,1 \mathrm{H}, \mathrm{CH}\right), 7.18-7.31$ (m, 9H, phenyl); ${ }^{13} \mathrm{C}$ NMR $\left.\delta 21.2\left(\mathrm{CH}_{3}\right), 24.1\left(\mathrm{C}\left(\mathrm{CH}_{3}\right)_{2}\right), 31.1\left(\mathrm{CH}_{2} \mathrm{Ph}\right), 34.0\left(\mathrm{CH}\left(\mathrm{CH}_{3}\right)_{2}\right)\right), 36.3$ $\left(\mathrm{C}(\mathrm{O}) \mathrm{CH}_{2}\right), 72.4(\mathrm{CH}), 126.3,126.7,128.4,128.5,128.6,139.1,140.7,148.6$ (phenyl), 172.3 $(C=\mathrm{O})$; HRMS calcd for $\mathrm{C}_{20} \mathrm{H}_{24} \mathrm{O}_{2} \mathrm{Na}[\mathrm{M}+\mathrm{Na}]^{+}$319.1673. Found: 319.1693 . The enantiomers were separated using GC (program A; $(S)-6 \mathbf{a}, t_{\mathrm{R}}=60.4 \mathrm{~min} ;(R)-6 \mathbf{a}, t_{\mathrm{R}}=60.7 \mathrm{~min}$ ).

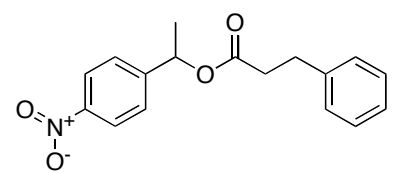

Dihydrocinnamic acid 1-(4-nitrophenyl)-ethyl ester (7a). Purification on silica gel (9:1 hexanes/EtOAc) to give a yellow solid $(2.64 \mathrm{~g}, 88 \%): \mathrm{mp} 46-48{ }^{\circ} \mathrm{C} ;{ }^{1} \mathrm{H}$ NMR $\delta 1.52\left(\mathrm{~d}, J=6.9,3 \mathrm{H}, \mathrm{CH}_{3}\right), 2.72$ $\left(\mathrm{m}, 2 \mathrm{H}, \mathrm{C}(\mathrm{O}) \mathrm{CH}_{2}\right), 2.98\left(\mathrm{t}, J=7.5,2 \mathrm{H}, \mathrm{CH}_{2} \mathrm{Ph}\right), 5.91(\mathrm{q}, J=6.6,1 \mathrm{H}$, $\mathrm{CH}$ ), 7.18-7.32 (m, 5H, phenyl), 7.39-8.20 (m, 4H, phenyl); ${ }^{13} \mathrm{C}$ NMR $\delta 22.3\left(\mathrm{CH}_{3}\right), 30.9$ $\left(\mathrm{CH}_{2} \mathrm{Ph}\right), 36.0\left(\mathrm{C}(\mathrm{O}) \mathrm{CH}_{2}\right), 71.4(\mathrm{CH}), 123.9,126.5,126.8,128.4,128.6,140.2,147.5,149.0$ (phenyl), $172.0(C=\mathrm{O})$; HRMS calcd for $\mathrm{C}_{17} \mathrm{H}_{17} \mathrm{NO}_{4} \mathrm{Na}[\mathrm{M}+\mathrm{Na}]^{+}$322.1055. Found: 322.1051 . The enantiomers were separated using GC (program A; $(S)-7 \mathbf{a}, t_{\mathrm{R}}=59.9 \mathrm{~min} ;(R)-7 \mathbf{a}, t_{\mathrm{R}}=60.8$ $\min )$.

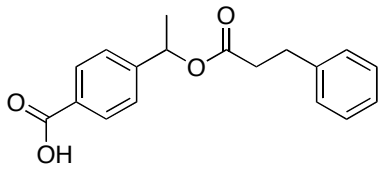

Dihydrocinnamic acid 1-(4-carboxylphenyl)-ethyl ester (8a). Purification on silica gel (95:5 to $90: 10$ hexanes/EtOAc) to give a white solid (316 g, 61\%): $\mathrm{mp} 74-76{ }^{\circ} \mathrm{C} ;{ }^{1} \mathrm{H}$ NMR $\delta 1.53$ (d, $J=6.9,3 \mathrm{H}, \mathrm{CH}_{3}$ ), $2.74\left(\mathrm{~m}, 2 \mathrm{H}, \mathrm{C}(\mathrm{O}) \mathrm{CH}_{2}\right), 2.98\left(\mathrm{t}, J=7.8,2 \mathrm{H}, \mathrm{CH}_{2} \mathrm{Ph}\right), 5.93(\mathrm{q}, J=6.9$, 
$1 \mathrm{H}, \mathrm{CH}), 7.19-7.33$ (m, 5H, phenyl), 7.37-8.10 (m, 4H, phenyl); ${ }^{13} \mathrm{C}$ NMR $\delta 22.3\left(\mathrm{CH}_{3}\right), 31.0$ $\left(\mathrm{CH}_{2} \mathrm{Ph}\right), 36.1\left(\mathrm{C}(\mathrm{O}) \mathrm{CH}_{2}\right), 71.9(\mathrm{CH}), 126.1,126.4,128.4,128.6,130.6,137.6,138.7,140.6$, 147.8 (phenyl), $171.8(C=\mathrm{O}), 172.2(\mathrm{COOH})$; HRMS calcd for $\mathrm{C}_{18} \mathrm{H}_{18} \mathrm{O}_{4} \mathrm{Na}[\mathrm{M}+\mathrm{Na}]^{+} 321.1102$.

Found: 321.1036. The enantiomers were separated uisng HPLC (Chiralpak AD-H column, 75:25 hexanes/EtOH, $\left.0.5 \mathrm{~mL} / \mathrm{min}, 254 \mathrm{~nm} ;(R)-8 \mathbf{a}, t_{\mathrm{R}}=17.5 \mathrm{~min} ;(S)-8 \mathbf{a}, t_{\mathrm{R}}=19.0 \mathrm{~min}\right)$.

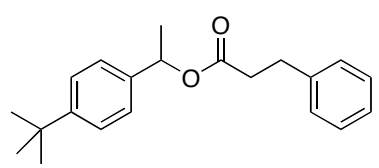

Dihydrocinnamic acid 1-(4-tert-butylphenyl) ethyl ester (9a). Purification on silica gel (100\% hexanes to $95: 5$ hexanes/EtOAc) to give a clear liquid $(1.01 \mathrm{~g}, 82 \%):{ }^{1} \mathrm{H}$ NMR $\delta 1.34\left(\mathrm{~s}, 9 \mathrm{H}, \mathrm{C}\left(\mathrm{CH}_{3}\right)_{3}\right), 1.52(\mathrm{~d}, J$ $\left.=6.6,3 \mathrm{H}, \mathrm{CH}_{3}\right), 2.67\left(\mathrm{~m}, 2 \mathrm{H}, \mathrm{C}(\mathrm{O}) \mathrm{CH}_{2}\right), 2.97\left(\mathrm{t}, J=7.8,2 \mathrm{H}, \mathrm{CH}_{2} \mathrm{Ph}\right)$,

$5.90(\mathrm{q}, J=6.6,1 \mathrm{H}, \mathrm{CH}), 7.18-7.39\left(\mathrm{~m}, 9 \mathrm{H}\right.$, phenyl); ${ }^{13} \mathrm{C}$ NMR $\delta 22.1\left(\mathrm{CH}_{3}\right), 31.1\left(\mathrm{CH}_{2} \mathrm{Ph}\right), 31.5$

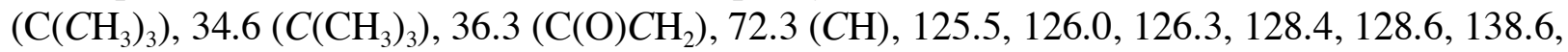
140.6, 150.9 (phenyl), $172.3(C=\mathrm{O})$; HRMS calcd for $\mathrm{C}_{21} \mathrm{H}_{26} \mathrm{O}_{2} \mathrm{Na}[\mathrm{M}+\mathrm{Na}]^{+}$333.1830. Found: 333.1852. The enantiomers were separated using GC (program A; $(S)-9 \mathbf{a}, t_{\mathrm{R}}=66.3 \mathrm{~min} ;(R)-\mathbf{9 a}$, $\left.t_{\mathrm{R}}=66.6 \mathrm{~min}\right)$.

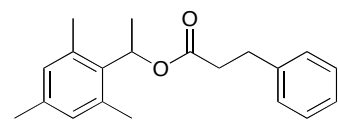

Dihydrocinnamic acid 1-(2,4,6-trimethylphenyl) ethyl ester (10a). Purification on silica gel (100\% hexanes to $99: 1$ hexanes/EtOAc) to give a clear liquid $(1.43 \mathrm{~g}, 70 \%)$ : ${ }^{1} \mathrm{H}$ NMR $\delta 1.54\left(\mathrm{~d}, J=6.9,3 \mathrm{H}, \mathrm{CH}_{3}\right)$, 2.27 (s, $\left.3 \mathrm{H}, p-\mathrm{CH}_{3}\right), 2.42\left(\mathrm{~s}, 6 \mathrm{H}, o-\mathrm{CH}_{3}\right), 2.66\left(\mathrm{~m}, 2 \mathrm{H}, \mathrm{C}(\mathrm{O}) \mathrm{CH}_{2}\right), 2.95$ (t, $\left.J=7.8,2 \mathrm{H}, \mathrm{CH}_{2} \mathrm{Ph}\right)$, 6.30 (q, $J=7.2,1 \mathrm{H}, \mathrm{CH}), 6.84$ (s, 2H, phenyl), 7.17-7.29 (m, 5H, phenyl); ${ }^{13} \mathrm{C}$ NMR $\delta 19.7$ $\left(\mathrm{CH}_{3}\right), 20.6\left(\mathrm{CH}_{3}\right), 20.9\left(\mathrm{CH}_{3}\right), 31.1\left(\mathrm{CH}_{2} \mathrm{Ph}\right), 36.2\left(\mathrm{C}(\mathrm{O}) \mathrm{CH}_{2}\right), 69.7(\mathrm{CH}), 126.4,128.4,128.6$, 130.1, 134.5, 136.1, 137.1, 140.7 (phenyl), $172.3(C=\mathrm{O})$; HRMS calcd for $\mathrm{C}_{20} \mathrm{H}_{24} \mathrm{O}_{2} \mathrm{Na}[\mathrm{M}+\mathrm{Na}]^{+}$ 319.1673. Found: 319.1409 . The enantiomers could not be separated using GC or HPLC.

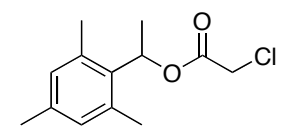

Chloroacetic acid 1-(2,4,6-trimethylphenyl) ethyl ester (10b). Purification on silica gel (100\% hexanes to $95: 5$ hexanes/EtOAc) to give a clear liquid (1.11 g, 90\%): ${ }^{1} \mathrm{H}$ NMR $\delta 1.63\left(\mathrm{~d}, J=6.9,3 \mathrm{H}, \mathrm{CH}_{3}\right), 2.26(\mathrm{~s}, 3 \mathrm{H}$, $\left.p-\mathrm{CH}_{3}\right), 2.45\left(\mathrm{~s}, 6 \mathrm{H}, o-\mathrm{CH}_{3}\right), 4.08\left(\mathrm{~d}, \mathrm{~J}=2.4, \mathrm{CH}_{2} \mathrm{Cl}\right), 6.37$ (q, $\left.\mathrm{J}=6.9,1 \mathrm{H}, \mathrm{CH}\right), 6.85(\mathrm{~s}, 2 \mathrm{H}$, phenyl); ${ }^{13} \mathrm{C}$ NMR $\delta 19.6\left(\mathrm{CH}_{3}\right), 20.5\left(\mathrm{CH}_{3}\right), 20.9\left(\mathrm{CH}_{3}\right), 41.2\left(\mathrm{CH}_{2} \mathrm{Cl}\right), 71.9(\mathrm{CH}), 130.2,133.5$, 136.1, 137.5 (phenyl), $166.7(C=\mathrm{O})$; HRMS calcd for $\mathrm{C}_{13} \mathrm{H}_{17}{ }^{35} \mathrm{ClO}_{2} \mathrm{Na}[\mathrm{M}+\mathrm{Na}]^{+} 263.0814$. Found: 263.0798. The enantiomers could not be separated using GC or HPLC.

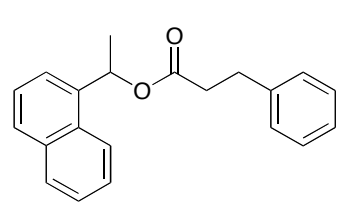

Dihydrocinnamic acid 1-(1-naphthyl) ethyl ester (11a).

Purification on silica gel (100\% hexanes to $98: 2$ hexanes/EtOAc) to give a clear liquid $(1.37 \mathrm{~g}, 75 \%)$ : ${ }^{1} \mathrm{H}$ NMR $\delta 1.69\left(\mathrm{~d}, J=6.6,3 \mathrm{H}, \mathrm{CH}_{3}\right), 2.74(\mathrm{~m}$, $\left.2 \mathrm{H}, \mathrm{C}(\mathrm{O}) \mathrm{CH}_{2}\right), 3.00\left(\mathrm{t}, J=7.8,2 \mathrm{H}, \mathrm{CH}_{2} \mathrm{Ph}\right), 6.67(\mathrm{q}, J=6.6,1 \mathrm{H}, \mathrm{CH})$, 7.20-8.10 (m, 12H, phenyl); ${ }^{13} \mathrm{C}$ NMR $\delta 21.8\left(\mathrm{CH}_{3}\right), 31.1\left(\mathrm{CH}_{2} \mathrm{Ph}\right), 36.2$ $\left(\mathrm{C}(\mathrm{O}) \mathrm{CH}_{2}\right), 69.7(\mathrm{CH}), 123.3,123.3,125.5,125.8,126.4,126.4,128.4,128.5,128.6,129.0$, $130.3,133.9,137.5,140.6$ (phenyl), $172.3(C=\mathrm{O})$; HRMS calcd for $\mathrm{C}_{21} \mathrm{H}_{20} \mathrm{O}_{2} \mathrm{Na}[\mathrm{M}+\mathrm{Na}]^{+}$ 327.1360. Found: 327.1304 . The enantiomers were separated using HPLC (Chiralcel OD column, 90:10 hexanes/EtOH, $0.75 \mathrm{~mL} / \mathrm{min}, 254 \mathrm{~nm} ;(R)-11 \mathrm{a}, t_{\mathrm{R}}=6.7 \mathrm{~min} ;(S)-11 \mathrm{a}, t_{\mathrm{R}}=7.4$ $\min )$.

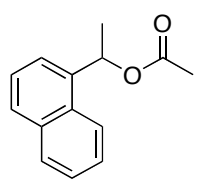

Acetic acid 1-(1-naphthyl) ethyl ester (11c). Purification on silica gel (100\% hexanes to $95: 5$ hexanes/EtOAc) to give a clear liquid $(860 \mathrm{mg}, 80 \%):{ }^{1} \mathrm{H}$ 
NMR $\delta 1.73\left(\mathrm{~d}, J=6.6,3 \mathrm{H}, \mathrm{CH}_{3}\right), 2.15\left(\mathrm{~s}, 3 \mathrm{H}, \mathrm{C}(\mathrm{O}) \mathrm{CH}_{3}\right), 6.68(\mathrm{q}, J=6.6,1 \mathrm{H}, \mathrm{CH}), 7.47-8.12$ (m, 12H, phenyl); ${ }^{13} \mathrm{C}$ NMR $\delta 21.5\left(\mathrm{CH}_{3}\right), 21.8\left(\mathrm{CH}_{3}\right), 69.5(\mathrm{CH}), 123.3,123.3,125.5,125.8$, 126.4, 128.6, 129.0, 130.4, 133.9, 137.5 (phenyl), $170.5(C=\mathrm{O})$; HRMS calcd for $\mathrm{C}_{14} \mathrm{H}_{14} \mathrm{O}_{2} \mathrm{Na}$ $[\mathrm{M}+\mathrm{Na}]^{+}$237.0891. Found: 237.0888 . The enantiomers were separated using HPLC (Chiralcel OD column, $90: 10$ hexanes $/ \mathrm{EtOH}, 0.75 \mathrm{~mL} / \mathrm{min}, 254 \mathrm{~nm} ;(R)-\mathbf{1 1 c}, t_{\mathrm{R}}=5.8 \mathrm{~min} ;(S)-\mathbf{1 1 c}, t_{\mathrm{R}}=6.8$ $\min )$.

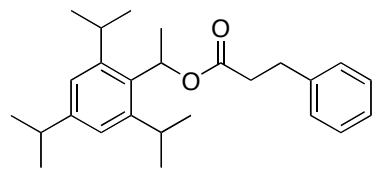

Dihydrocinnamic acid 1-(2,4,6-triisopropylphenyl) ethyl ester (12b). Purification on silica gel (100\% hexanes to 98:2 hexanes/EtOAc) to give a white solid $(819 \mathrm{mg}, 87 \%): \mathrm{mp} 80-82{ }^{\circ} \mathrm{C} ;{ }^{1} \mathrm{H}$ NMR $\delta 1.24(\mathrm{~d}, J$ $\left.=6.6,6 \mathrm{H}, \mathrm{C}\left(\mathrm{CH}_{3}\right)_{2}\right), 1.26\left(\mathrm{~d}, J=6.9,6 \mathrm{H}, \mathrm{C}\left(\mathrm{CH}_{3}\right)_{2}\right), 1.30(\mathrm{~d}, J=6.6,6 \mathrm{H}$, $\left.\mathrm{C}\left(\mathrm{CH}_{3}\right)_{2}\right), 1.69\left(\mathrm{~d}, J=6.9,3 \mathrm{H}, \mathrm{CH}_{3}\right), 2.63\left(\mathrm{~m}, 2 \mathrm{H}, \mathrm{C}(\mathrm{O}) \mathrm{CH}_{2}\right), 2.88$ (hept, $J=6.9,1 \mathrm{H}$, $\left.\mathrm{CH}\left(\mathrm{CH}_{3}\right)_{2}\right), 2.97\left(\mathrm{t}, J=7.8,2 \mathrm{H}, \mathrm{CH}_{2} \mathrm{Ph}\right), 3.50$ (br m, $\left.2 \mathrm{H}, 2 \mathrm{x} \mathrm{CH}\left(\mathrm{CH}_{3}\right)_{2}\right), 6.56(\mathrm{q}, J=6.9,1 \mathrm{H}$, $\mathrm{CH}$ ), 7.04 (s, 2H, phenyl), 7.19-7.32 (m, 5H, phenyl); ${ }^{13} \mathrm{C} \mathrm{NMR} \delta 22.2,24.0,24.4,24.9\left(\mathrm{CH}_{3}\right)$, $29.5\left(o-C H\left(\mathrm{CH}_{3}\right)_{2}\right), 31.2\left(\mathrm{CH}_{2} \mathrm{Ph}\right), 34.2\left(p-\mathrm{CH}\left(\mathrm{CH}_{3}\right)_{2}\right), 36.5\left(\mathrm{C}(\mathrm{O}) \mathrm{CH}_{2}\right), 68.4(\mathrm{CH}), 121.2,126.4$, 128.4, 128.6, 132.2, 140.6, 148.2 (phenyl), $172.4(C=\mathrm{O})$; HRMS calcd for $\mathrm{C}_{26} \mathrm{H}_{36} \mathrm{O}_{2} \mathrm{Na}[\mathrm{M}+\mathrm{Na}]^{+}$ 403.2613. Found: 403.2461. The enantiomers could not be separated using GC or HPLC.

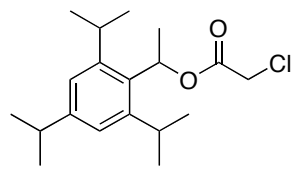

Chloroacetic acid 1-(2,4,6-triisopropylphenyl) ethyl ester (12b). Purification on silica gel (100\% hexanes to $98: 2$ hexanes/EtOAc) to give a white solid (562 mg, 86\%): $\mathrm{mp} \mathrm{47-49}{ }^{\circ} \mathrm{C} ;{ }^{1} \mathrm{H} \mathrm{NMR} \delta 1.25(\mathrm{~d}, J=6.9,6 \mathrm{H}$, $\left.\mathrm{C}\left(\mathrm{CH}_{3}\right)_{2}\right), 1.26\left(\mathrm{~d}, J=6.9,6 \mathrm{H}, \mathrm{C}\left(\mathrm{CH}_{3}\right)_{2}\right), 1.31\left(\mathrm{~d}, J=6.9,6 \mathrm{H}, \mathrm{C}\left(\mathrm{CH}_{3}\right)_{2}\right), 1.69$ (d, $\left.J=6.9,3 \mathrm{H}, \mathrm{CH}_{3}\right), 2.92$ (hept, $\left.J=6.9,1 \mathrm{H}, \mathrm{CH}\left(\mathrm{CH}_{3}\right)_{2}\right), 3.50$ (br m, $\left.2 \mathrm{H}, 2 \times \mathrm{CH}\left(\mathrm{CH}_{3}\right)_{2}\right), 4.07$ $\left(\mathrm{d}, J=6.6,2 \mathrm{H}, \mathrm{C}(\mathrm{O}) \mathrm{CH}_{2} \mathrm{Cl}\right), 6.62(\mathrm{q}, J=6.9,1 \mathrm{H}, \mathrm{CH}), 7.04\left(\mathrm{~s}, 2 \mathrm{H}\right.$, phenyl); ${ }^{13} \mathrm{C} \mathrm{NMR} \delta 22.2$, 23.9, 24.0, 24.3, $24.9\left(\mathrm{CH}_{3}\right), 29.5\left(o-\mathrm{CH}\left(\mathrm{CH}_{3}\right)_{2}\right), 34.2\left(p-\mathrm{CH}\left(\mathrm{CH}_{3}\right)_{2}\right), 41.3\left(\mathrm{CH}_{2} \mathrm{Cl}\right), 70.8(\mathrm{CH})$, 121.3, 131.3, 148.6 (phenyl), $166.8(C=\mathrm{O})$; HRMS calcd for $\mathrm{C}_{19} \mathrm{H}_{29}{ }^{35} \mathrm{ClO}_{2} \mathrm{Na}[\mathrm{M}+\mathrm{Na}]^{+} 347.1753$. Found: 347.1645. The enantiomers could not be separated using GC or HPLC.

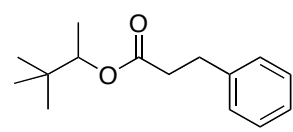

Dihydrocinnamic acid 1,2,2-trimethyl-propyl ester (13a). Purification on silica gel (100\% hexanes to $99: 1$ hexanes/EtOAc) to give a clear liquid (866 mg, 74\%): ${ }^{1} \mathrm{H}$ NMR $\delta 0.88\left(\mathrm{~s}, 9 \mathrm{H}, \mathrm{C}\left(\mathrm{CH}_{3}\right)_{3}\right), 1.11(\mathrm{~d}, J=$ 6.6, 3H, $\left.\mathrm{CH}_{3}\right), 2.65\left(\mathrm{~m}, 2 \mathrm{H}, \mathrm{C}(\mathrm{O}) \mathrm{CH}_{2}\right), 2.98\left(\mathrm{t}, J=7.8,2 \mathrm{H}, \mathrm{CH}_{2} \mathrm{Ph}\right), 4.70(\mathrm{q}, J=6.6,1 \mathrm{H}, \mathrm{CH})$,

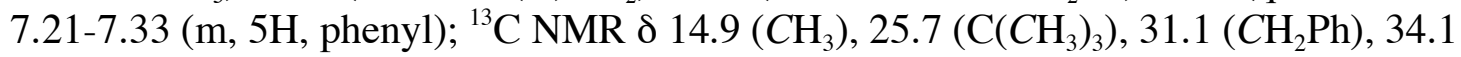
$\left(C\left(\mathrm{CH}_{3}\right)_{3}\right), 36.3\left(\mathrm{C}(\mathrm{O}) \mathrm{CH}_{2}\right), 77.7(\mathrm{CH}), 126.3,128.4,128.6,140.7$ (phenyl), $172.6(\mathrm{C}=\mathrm{O})$; HRMS calcd for $\mathrm{C}_{15} \mathrm{H}_{22} \mathrm{O}_{2} \mathrm{Na}[\mathrm{M}+\mathrm{Na}]^{+}$257.1517. Found: 257.1156. The enantiomers were separated using GC (program $\mathbf{A} ;(R$ or $S)-\mathbf{1 3 a}, t_{\mathrm{R}}=31.1 \mathrm{~min} ;(R$ or $\left.S)-\mathbf{1 3 a}, t_{\mathrm{R}}=31.2 \mathrm{~min}\right)$.

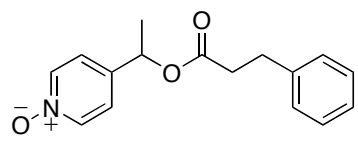

Dihydrocinnamic acid 1-(1-oxy-pyridin-4-yl)-ethyl ester (5a). $m$-CPBA $(77 \%, 670 \mathrm{mg}, 2.96 \mathrm{mmol})$ was added portion-wise to a stirred solution of $2 \mathbf{a}(757 \mathrm{mg}, 2.99 \mathrm{mmol})$ in $\mathrm{CH}_{2} \mathrm{Cl}_{2}(25 \mathrm{~mL})$ at $0^{\circ} \mathrm{C} .{ }^{5}$ The ice bath was removed and the reaction solution was stirred overnight at RT. The solution was concentrated in vacuo and the crude residue was purified on silica gel $\left(95: 5 \mathrm{CHCl}_{3} / \mathrm{MeOH}\right)$ to give a viscous, clear liquid (710 mg, 89\%): ${ }^{1} \mathrm{H}$ NMR $\delta 1.48\left(\mathrm{~d}, J=6.6,3 \mathrm{H}, \mathrm{CH}_{3}\right), 2.72(\mathrm{~m}, 2 \mathrm{H}$, $\left.\mathrm{C}(\mathrm{O}) \mathrm{CH}_{2}\right), 2.97\left(\mathrm{t}, J=7.5,2 \mathrm{H}, \mathrm{CH}_{2} \mathrm{Ph}\right), 5.79(\mathrm{q}, J=6.6,1 \mathrm{H}, \mathrm{CH}), 7.08(\mathrm{~d}, J=6.9,2 \mathrm{H}$, pyridyl), 7.18-7.32 (m, 7H, phenyl), 8.16 (d, $J=6.9,2 \mathrm{H}$, pyridyl); ${ }^{13} \mathrm{C} \mathrm{NMR} \delta 21.6\left(\mathrm{CH}_{3}\right), 31.8\left(\mathrm{CH}_{2} \mathrm{Ph}\right)$, $36.6\left(\mathrm{C}(\mathrm{O}) \mathrm{CH}_{2}\right), 71.3(\mathrm{CH}), 125.4,127.4,129.4,129.5,140.2,141.6,146.2$, (aromatic), 173.4 
$(C=\mathrm{O})$; HRMS calcd for $\mathrm{C}_{16} \mathrm{H}_{18} \mathrm{NO}_{3}[\mathrm{M}+\mathrm{H}]^{+}$272.1292. Found: 272.1298. The enantiomers were separated using GC (program B $;(S)-5 \mathbf{a}, t_{\mathrm{R}}=59.5 \mathrm{~min} ;(R)-\mathbf{5 a}, t_{\mathrm{R}}=60.2 \mathrm{~min}$ ).

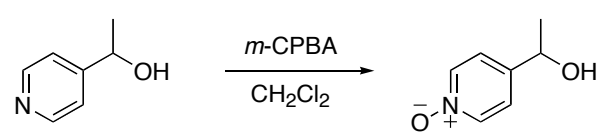

1-(1-Oxy-pyridin-4-yl)-ethanol (5). ${ }^{6} \mathrm{~m}$-CPBA $(77 \%, 1.82 \mathrm{~g}, 8.13 \mathrm{mmol})$ was added portion-wise to a stirred solution of 1-(pyrid-4-yl) ethanol (1.03 g, 8.13

mmol) in $\mathrm{CH}_{2} \mathrm{Cl}_{2}(50 \mathrm{~mL})$ at $0^{\circ} \mathrm{C} .{ }^{5}$ The ice bath was removed and the reaction solution was stirred overnight at RT. The solution was concentrated in vacuo and the crude residue was purified on silica gel $\left(4: 1 \mathrm{CHCl}_{3} / \mathrm{MeOH}\right)$ to give a white, hygroscopic solid $(1.07 \mathrm{~g}, 95 \%): \mathrm{mp} 79-81{ }^{\circ} \mathrm{C} ;{ }^{1} \mathrm{H}$ NMR $\delta 1.49\left(\mathrm{~d}, J=6.6,3 \mathrm{H}, \mathrm{CH}_{3}\right), 4.87(\mathrm{q}, J=6.6,1 \mathrm{H}, \mathrm{CH}), 7.26(\mathrm{~d}, J=6.6,2 \mathrm{H}$, pyridyl) 8.01 $\left(\mathrm{d}, J=6.9,2 \mathrm{H}\right.$, pyridyl); ${ }^{13} \mathrm{C} \mathrm{NMR}\left(\mathrm{CD}_{3} \mathrm{OD}\right) \delta 24.9\left(\mathrm{CH}_{3}\right), 68.4(\mathrm{CH}), 124.9,140.0,151.7$ (pyridyl); HRMS calcd for $\mathrm{C}_{7} \mathrm{H}_{10} \mathrm{NO}_{2}[\mathrm{M}+\mathrm{H}]^{+}$140.0717. Found: 140.0710. The enantiomers were separated using GC (program B; $(R)-\mathbf{4}, t_{\mathrm{R}}=20.7 \mathrm{~min} ;(S)-\mathbf{4}, t_{\mathrm{R}}=21.0 \mathrm{~min}$ ).

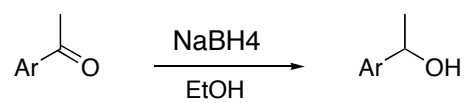

Synthesis of Racemic Secondary Alcohols 4, 6-10. General Procedure. $\mathrm{NaBH}_{4}(555 \mathrm{mg}, 15 \mathrm{mmol})$ was added portion-wise to a stirred solution of ketone $(10 \mathrm{mmol})$ in $\mathrm{EtOH}(10$ $\mathrm{mL})$ at $0^{\circ} \mathrm{C}$. The ice bath was removed and the reaction solution was stirred for $3 \mathrm{~h}$ at RT. The solution was cooled to $0{ }^{\circ} \mathrm{C}$ and quenched with $1 \mathrm{~N} \mathrm{HCl}(10 \mathrm{~mL})$ and extracted with $\mathrm{CH}_{2} \mathrm{Cl}_{2}(3 \mathrm{x}$ $20 \mathrm{~mL}$ ). The combined organic layers were washed with sat. $\mathrm{NaHCO}_{3}(25 \mathrm{~mL})$ (except 8), sat. $\mathrm{NaCl}(25 \mathrm{~mL})$ and dried over $\mathrm{Na}_{2} \mathrm{SO}_{4}$. The organic layer was concentrated in vacuo to give the crude alcohol. The relevant analytical data are given below:

1-(4-Trifluorophenyl)-ethanol (4). ${ }^{7}$ Obtained as clear liquid $(1.74 \mathrm{~g}$,

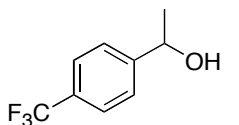
97\%): ${ }^{1} \mathrm{H}$ NMR $\delta 1.52\left(\mathrm{~d}, J=6.3,3 \mathrm{H}, \mathrm{CH}_{3}\right), 1.87$ (br s, $\left.1 \mathrm{H}, \mathrm{OH}\right), 4.99(\mathrm{q}, J=6.6$, $1 \mathrm{H}, \mathrm{CH}), 7.50-7.64\left(\mathrm{~m}, 4 \mathrm{H}\right.$, phenyl); ${ }^{13} \mathrm{C}$ NMR $\delta 25.4\left(\mathrm{CH}_{3}\right), 69.8(\mathrm{CH}), 125.5(\mathrm{q}$, $\left.C \mathrm{~F}_{3}\right), 125.6,125.7,149.8$ (phenyl). The enantiomers were separated using GC (program $\mathbf{C} ;(R)$ 4, $\left.t_{\mathrm{R}}=10.6 \mathrm{~min} ;(S)-\mathbf{4}, t_{\mathrm{R}}=10.9 \mathrm{~min}\right)$.

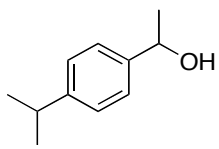

1-(4-Isopropylphenyl)-ethanol (6). Obtained as clear liquid (2.82 $\mathrm{g}$, $85 \%):{ }^{1} \mathrm{H} \mathrm{NMR} \delta 1.27\left(\mathrm{~d}, J=6.9,6 \mathrm{H}, \mathrm{CH}\left(\mathrm{CH}_{3}\right)_{2}\right), 1.51\left(\mathrm{~d}, J=6.3,3 \mathrm{H}, \mathrm{CH}_{3}\right)$, 1.86 (br s, $1 \mathrm{H}, \mathrm{OH}), 2.93$ (hept, $\left.J=6.9,1 \mathrm{H}, \mathrm{CH}\left(\mathrm{CH}_{3}\right)_{2}\right), 4.89$ (q, $J=6.3,1 \mathrm{H}$, $\mathrm{CH}), 7.23-7.34\left(\mathrm{~m}, 4 \mathrm{H}\right.$, phenyl); ${ }^{13} \mathrm{C} \mathrm{NMR} \delta 24.1\left(\mathrm{CH}_{3}\right), 25.0\left(\mathrm{C}_{\left.\left(\mathrm{CH}_{3}\right)_{2}\right), 33.9}\right.$ $\left.\left(\mathrm{CH}\left(\mathrm{CH}_{3}\right)_{2}\right)\right), 70.3(\mathrm{CH}), 125.6,126.6,143.4,148.2$ (phenyl); HRMS calcd for $\mathrm{C}_{11} \mathrm{H}_{16} \mathrm{ONa}$ $[\mathrm{M}+\mathrm{Na}]^{+}$187.1098. Found: 187.1100 . The enantiomers were separated using GC (program A; $\left.(R)-6, t_{\mathrm{R}}=24.9 \mathrm{~min} ;(S)-6, t_{\mathrm{R}}=25.4 \mathrm{~min}\right)$.

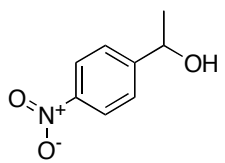

1-(4-Nitrophenyl)-ethanol (7). ${ }^{8}$ Obtained as yellow liquid $(2.98 \mathrm{~g}, 90 \%)$ : ${ }^{1} \mathrm{H}$ NMR $\delta 1.54$ (d, $\left.J=6.6,3 \mathrm{H}, \mathrm{CH}_{3}\right), 1.97$ (br s, $\left.1 \mathrm{H}, \mathrm{OH}\right), 5.04(\mathrm{q}, J=6.6,1 \mathrm{H}$, $\mathrm{CH}), 7.55-8.24\left(\mathrm{~m}, 4 \mathrm{H}\right.$, phenyl); ${ }^{13} \mathrm{C}$ NMR $\delta 25.6\left(\mathrm{CH}_{3}\right), 69.6(\mathrm{CH}), 123.8,126.2$, 147.2, 153.2 (phenyl). The enantiomers were separated using GC (program A; $\left.(R)-7, t_{\mathrm{R}}=42.1 \mathrm{~min} ;(S)-7, t_{\mathrm{R}}=43.1 \mathrm{~min}\right)$.

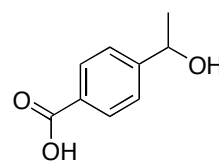

4-(1-Hydroxyethyl)-benzoic acid (8). Obtained as white solid (1.49 g, 90\%): $\mathrm{mp} 137-139{ }^{\circ} \mathrm{C} ;{ }^{1} \mathrm{H} \mathrm{NMR}\left(\mathrm{CD}_{3} \mathrm{OD}\right) \delta 1.53\left(\mathrm{~d}, J=6.3,3 \mathrm{H}, \mathrm{CH}_{3}\right), 4.92(\mathrm{~m}$, $1 \mathrm{H}, \mathrm{CH}), 7.49-8.04$ (m, 4H, phenyl); ${ }^{13} \mathrm{C} \mathrm{NMR}\left(\mathrm{CD}_{3} \mathrm{OD}\right) \delta 26.4\left(\mathrm{CH}_{3}\right), 71.3$ 
$(C \mathrm{H}), 127.3,131.4,131.7,154.0$ (phenyl), $170.7(\mathrm{COOH})$; HRMS calcd for $\mathrm{C}_{9} \mathrm{H}_{10} \mathrm{O}_{3} \mathrm{Na}[\mathrm{M}+\mathrm{Na}]^{+}$ 189.0527. Found: 189.0264. The enantiomers were separated using HPLC (Chiralpak AD-H column, 75:25 hexanes/EtOH, $\left.0.5 \mathrm{~mL} / \mathrm{min}, 254 \mathrm{~nm} ;(R)-8, t_{\mathrm{R}}=13.9 \mathrm{~min} ;(S)-8, t_{\mathrm{R}}=15.3 \mathrm{~min}\right)$.

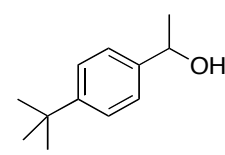

1-(4-tert-Butylphenyl)-ethanol (9). Obtained as white solid (1.74 g, 97\%): $\mathrm{mp} 60-62{ }^{\circ} \mathrm{C} ;{ }^{1} \mathrm{H}$ NMR $\delta 1.34\left(\mathrm{~s}, 9 \mathrm{H}, \mathrm{C}\left(\mathrm{CH}_{3}\right)_{3}\right), 1.52\left(\mathrm{~d}, J=6.0,3 \mathrm{H}, \mathrm{CH}_{3}\right)$, $4.90(\mathrm{q}, J=6.3,1 \mathrm{H}, \mathrm{CH}), 7.32-7.42\left(\mathrm{~m}, 4 \mathrm{H}\right.$, phenyl); ${ }^{13} \mathrm{C} \mathrm{NMR} \delta 25.0\left(\mathrm{CH}_{3}\right)$, $31.5\left(\mathrm{C}\left(\mathrm{CH}_{3}\right)_{3}\right), 34.6\left(\mathrm{C}\left(\mathrm{CH}_{3}\right)_{3}\right), 70.3(\mathrm{CH}), 125.3,125.5,142.9,150.5$ (phenyl); HRMS calcd for $\mathrm{C}_{12} \mathrm{H}_{18} \mathrm{ONa}[\mathrm{M}+\mathrm{Na}]^{+} 201.1255$. Found: 201.1007. The enantiomers were separated using GC (program $\left.\mathbf{A} ;(R)-\mathbf{9}, t_{\mathrm{R}}=28.1 \mathrm{~min} ;(S)-\mathbf{9}, t_{\mathrm{R}}=28.4 \mathrm{~min}\right)$.

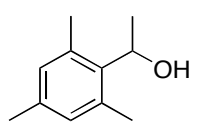

1-(2,4,6-trimethylphenyl)-ethanol (10). ${ }^{7}$ Obtained as white solid (3.01 g, 92\%): $\mathrm{mp} 66-68{ }^{\circ} \mathrm{C} ;{ }^{1} \mathrm{H}$ NMR $\delta 1.53\left(\mathrm{~d}, J=6.6,3 \mathrm{H}, \mathrm{CH}_{3}\right), 2.25\left(\mathrm{~s}, 3 \mathrm{H}, p-\mathrm{CH}_{3}\right)$, $2.42\left(\mathrm{~s}, 6 \mathrm{H}, o-\mathrm{CH}_{3}\right), 5.37(\mathrm{q}, J=6.9,1 \mathrm{H}, \mathrm{CH}), 6.82\left(\mathrm{~s}, 2 \mathrm{H}\right.$, phenyl); ${ }^{13} \mathrm{C}$ NMR $\delta$ $20.6\left(\mathrm{CH}_{3}\right), 20.8\left(\mathrm{CH}_{3}\right), 21.6\left(\mathrm{CH}_{3}\right), 67.5(\mathrm{CH}), 130.2,135.8,136.5,137.8$ (phenyl). The enantiomers were separated using GC (program $\left.\mathbf{C} ;(R)-\mathbf{1 0}, t_{\mathrm{R}}=12.9 \mathrm{~min} ;(S)-\mathbf{1 0}, t_{\mathrm{R}}=13.3 \mathrm{~min}\right)$.

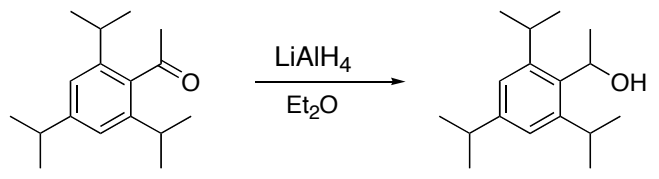

\section{Synthesis of 1-(2,4,6-triisopropylphenyl)-} ethanol (12). ${ }^{9} 2,4,6$-Triisopropylacetophenone $(2.46 \mathrm{~g}$, $10 \mathrm{mmol})$ in anhydrous $\mathrm{Et}_{2} \mathrm{O}(10 \mathrm{~mL})$ was added dropwise to a stirred slurry of $\mathrm{LiAlH}_{4}(570 \mathrm{mg}, 15 \mathrm{mmol})$ in anhydrous $\mathrm{Et}_{2} \mathrm{O}(20 \mathrm{~mL})$ at $0^{\circ} \mathrm{C}$. The ice bath was removed and the reaction solution was stirred for $3 \mathrm{~h}$ at RT. The solution was then cooled to $0^{\circ} \mathrm{C}$ and quenched with $1 \mathrm{~N} \mathrm{HCl}(10 \mathrm{~mL})$ and extracted with EtOAc $(3 \times 25 \mathrm{~mL})$. The combined organic layers were washed with sat. $\mathrm{NaHCO}_{3}$ $(25 \mathrm{~mL})$, sat. $\mathrm{NaCl}(25 \mathrm{~mL})$ and dried over $\mathrm{Na}_{2} \mathrm{SO}_{4}$. The organic layer was concentrated in vacuo to give a white solid $(1.62 \mathrm{~g}, 65 \%)$ : $\mathrm{mp} 85-87{ }^{\circ} \mathrm{C}$ (lit. $\left.{ }^{9} 84-85{ }^{\circ} \mathrm{C}\right) ;{ }^{1} \mathrm{H}$ NMR $\delta 1.27(\mathrm{~d}, J=6.9$, $\left.12 \mathrm{H}, 2 \times \mathrm{C}\left(\mathrm{CH}_{3}\right)_{2}\right), 1.29$ (d, $\left.J=6.9,6 \mathrm{H}, \mathrm{C}\left(\mathrm{CH}_{3}\right)_{2}\right), 1.63$ (d, J=6.6, 3H, $\left.\mathrm{CH}_{3}\right), 1.76$ (br s, $1 \mathrm{H}$, $\mathrm{OH}), 2.88$ (hept, $\left.J=6.9,1 \mathrm{H}, \mathrm{CH}\left(\mathrm{CH}_{3}\right)_{2}\right), 3.61\left(\mathrm{~m}, 2 \mathrm{H}, 2 \times \mathrm{CH}\left(\mathrm{CH}_{3}\right)_{2}\right), 5.55(\mathrm{q}, J=6.9,1 \mathrm{H}, \mathrm{CH})$, 7.05 (s, 2H, phenyl); ${ }^{13} \mathrm{C}$ NMR $\delta 23.7,24.0,24.1,24.7,24.8\left(\mathrm{CH}_{3}\right), 29.1\left(o-C H\left(\mathrm{CH}_{3}\right)_{2}\right), 34.2(p-$ $\left.\mathrm{CH}\left(\mathrm{CH}_{3}\right)_{2}\right), 66.2(\mathrm{CH}), 121.1,122.2,136.0,147.7$ (phenyl). The enantiomers were separated using GC (program C; $\left.(R)-\mathbf{1 1}, t_{\mathrm{R}}=14.2 \mathrm{~min} ;(S)-\mathbf{1 1}, t_{\mathrm{R}}=14.5 \mathrm{~min}\right)$.

Determination of Absolute Configuration. Enantiopure alcohols $(R)-1,(R)-2$ and $(R)$ 11 were purchased from Sigma-Aldrich Corporation. Absolute configurations of alcohols 3, 4, 7 and 10 were assigned by similarity in the order of elution of enantiomers of previously reported GC analyses. ${ }^{7,8}$ Enantiopure $(R)-4$ was synthesized via $m$-CPBA oxidation ${ }^{5}$ of $(R)-3$, as described above for (+/-)-4. Absolute configurations of alcohols 6,8 and 9 were assigned using the configurational correlation model for the corresponding $(R)$-MTPA derivatives, which were synthesized using $(S)-(+)-\alpha$-methoxy- $\alpha$-(trifluoromethyl)-phenylacetyl chloride in pyridine. ${ }^{10}$ Enantiopure $(S)-6$ and $(S)-9$ were prepared via PCL-catalyzed acylation of racemic alcohol with vinyl acetate and $(S)-\mathbf{8}$ was prepared by PCL-catalyzed hydrolysis of $\mathbf{8 a}$ followed by hydrolysis of the remaining starting material. The relevant analytical data are below:

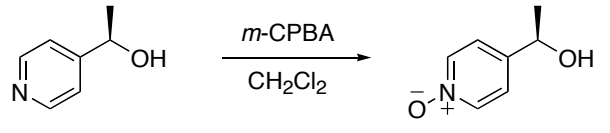

(R)-1-(1-oxy-pyridin-4-yl)-ethanol $((\boldsymbol{R})-5){ }^{6}$

Obtained as low-melting white solid $(265 \mathrm{mg}, 95 \%, 98 \%$ ee): ${ }^{1} \mathrm{H}$ NMR $\delta 1.46\left(\mathrm{~d}, J=6.6,3 \mathrm{H}, \mathrm{CH}_{3}\right), 4.85(\mathrm{q}, J=6.6$, 
1H, CH), $7.26\left(\mathrm{~d}, J=7.2,2 \mathrm{H}\right.$, pyridyl) $8.00\left(\mathrm{~d}, J=7.2,2 \mathrm{H}\right.$, pyridyl); ${ }^{13} \mathrm{C} \mathrm{NMR}\left(\mathrm{CD}_{3} \mathrm{OD}\right) \delta 24.9$ $\left(\mathrm{CH}_{3}\right), 68.4(\mathrm{CH}), 124.9,140.0,151.7$ (pyridyl); HRMS calcd for $\mathrm{C}_{7} \mathrm{H}_{10} \mathrm{NO}_{2}[\mathrm{M}+\mathrm{H}]^{+} 140.0717$. Found: 140.0710 .

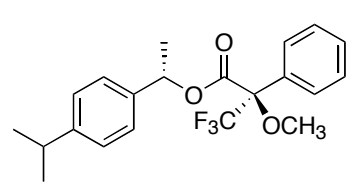

$(\boldsymbol{R}, \boldsymbol{S})$-1-(4-Isopropylphenyl)-ethyl MTPA ester. ${ }^{1} \mathrm{H}$ NMR $\delta 1.26$ $\left(\mathrm{d}, J=6.9,6 \mathrm{H}, \mathrm{CH}\left(\mathrm{CH}_{3}\right)_{2}, S\right.$-enantiomer $), 1.27$ (d, $J=6.9,6 \mathrm{H}, \mathrm{CH}\left(\mathrm{CH}_{3}\right)_{2}$, $R$-enantiomer), 1.59 (d, $\left.J=6.6,3 \mathrm{H}, \mathrm{CH}_{3}\right), R$-enantiomer), $1.65(\mathrm{~d}, J=6.6$, $3 \mathrm{H}, \mathrm{CH}_{3}$ ), $\mathrm{S}$-enantiomer), 2.91 (hept, $\left.J=6.6,1 \mathrm{H}, \mathrm{CH}\left(\mathrm{CH}_{3}\right)_{2}\right), 3.50$ (q, $J=$ $1.5,3 \mathrm{H}, \mathrm{OCH}_{3}, R$-enantiomer), 3.58 (q, $J=1.5,3 \mathrm{H}, \mathrm{OCH}_{3}, S$-enantiomer), 6.10 (q, $J=6.6,1 \mathrm{H}$, $\mathrm{CH}, \mathrm{S}$-enantiomer), 6.14 (q, $J=6.6,1 \mathrm{H}, \mathrm{CH}, R$-enantiomer), 7.15-7.43 (m, 9H, phenyl).

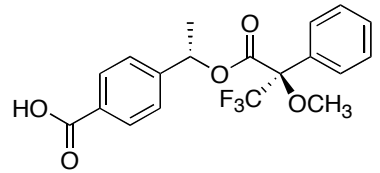

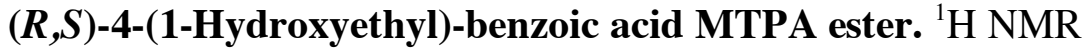
$\delta 1.61\left(\mathrm{~d}, J=6.6,3 \mathrm{H}, \mathrm{CH}_{3}, R\right.$-enantiomer), $1.66\left(\mathrm{~d}, J=6.6,3 \mathrm{H}, \mathrm{CH}_{3}, S\right.$ enantiomer), 3.50 (q, $J=1.5,3 \mathrm{H}, \mathrm{OCH}_{3}, R$-enantiomer), 3.59 (q, $J=1.5$, $3 \mathrm{H}, \mathrm{OCH}_{3}, \mathrm{~S}$-enantiomer), 6.14 (q, $J=6.6,1 \mathrm{H}, \mathrm{CH}, \mathrm{S}$-enantiomer), 6.18 (q, $J=6.6,1 \mathrm{H}, \mathrm{CH}, R$-enantiomer), 7.21-8.13 (m, 9H, phenyl).

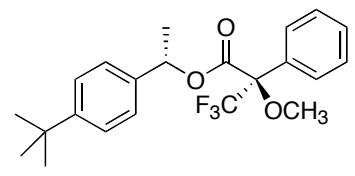

$(\boldsymbol{R}, \boldsymbol{S})$-1-(4-tert-butylphenyl)-ethyl MTPA ester. ${ }^{1} \mathrm{H}$ NMR $\delta 1.33$ (s, $9 \mathrm{H}, \mathrm{C}\left(\mathrm{CH}_{3}\right)_{3}, S$-enantiomer), 1.34 (s, 9H, $\left.\mathrm{CH}_{3}\right)_{3}, R$-enantiomer), 1.59 (d, $J=6.6,3 \mathrm{H}, \mathrm{CH}_{3}, R$-enantiomer), 1.65 (d, $J=6.6,3 \mathrm{H}, \mathrm{CH}_{3}, S$ enantiomer), $3.50\left(\mathrm{q}, J=1.5,3 \mathrm{H}, \mathrm{OCH}_{3}, R\right.$-enantiomer), $3.58(\mathrm{q}, J=1.5$, $3 \mathrm{H}, \mathrm{OCH}_{3}, S$-enantiomer), 6.10 (q, $J=6.6,1 \mathrm{H}, \mathrm{CH}, S$-enantiomer), 6.14 (q, $J=6.6,1 \mathrm{H}$, $\mathrm{CH}\left(\mathrm{CH}_{3}\right), R$-enantiomer), 7.19-7.43 (m, 9H, phenyl).
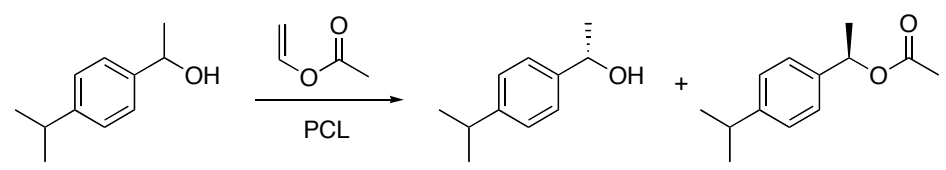

(S)-1-(4-isopropylphenyl)ethanol ((S)-6). Racemic 6 (26 mg), PCL (20 mg) and $4 \AA$ molecular sieves were stirred together in vinyl acetate $(1 \mathrm{~mL})$ at RT. After $72 \mathrm{~h}$, the reaction solution was concentrated and product and starting material were separated on silica gel (100\% hexanes to 9:1 hexanes/EtOAc). Unreacted starting material was isolated as a clear liquid (12 $\mathrm{mg}, 46 \%, 99 \%$ ee).

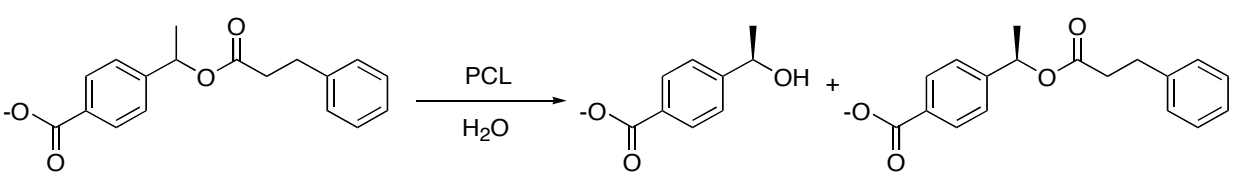

(S) 4-(1Hydroxyethyl)benzoic acid $((S)-8)$. A solution of racemic 8a $(61 \mathrm{mg})$ in $\mathrm{CH}_{3} \mathrm{CN}(200 \mu \mathrm{L})$ was added to a solution of PCL (50 mg) in $50 \mathrm{mM}$ BES $(\mathrm{pH} 7.2,1.8 \mathrm{~mL}$ ) and stirred at RT. After $72 \mathrm{~h}$, the reaction solution was acidified with $1 \mathrm{~N} \mathrm{HCl}$ $(1 \mathrm{~mL})$ and extracted with EtOAc $(3 \times 2 \mathrm{~mL})$. The combined organic layers were concentrated and product and starting material were separated on silica gel (4:1 hexanes/EtOAc to 100\% EtOAc). Remaining starting material was hydrolyzed in $1 \mathrm{~N} \mathrm{KOH}\left(1: 1 \mathrm{H}_{2} \mathrm{O} / \mathrm{EtOH}\right)$ to give a white solid (21 mg, 63\%, 15\% ee).

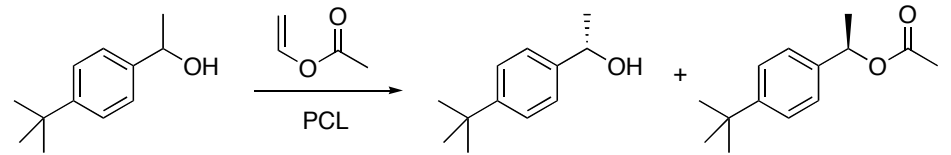

(S)-1-(4-tert-butylphenyl)ethanol ((S)-9). Racemic 9 (28 mg), PCL (20 mg) and $4 \AA$ Å molecular 
sieves were stirred together in vinyl acetate $(1 \mathrm{~mL})$ at RT. After $72 \mathrm{~h}$, the reaction solution was concentrated and product and starting material were separated on silica gel (100\% hexanes to 9:1 hexanes/EtOAc). Unreacted starting material was isolated as a clear liquid (13 mg, 46\%, 99\% ee).

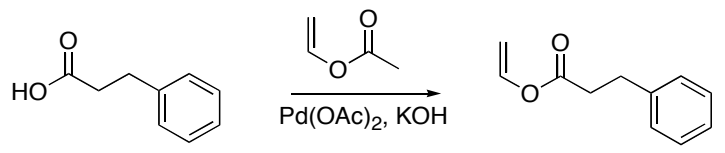

\author{
Dihydrocinnamic vinyl ester. ${ }^{11}$ A solution \\ of dihydrocinnamic acid $(1.05 \mathrm{~g}, 7 \mathrm{mmol})$, \\ $\mathrm{Pd}(\mathrm{OAc})_{2}(313 \mathrm{mg}, 0.2 \mathrm{eq}),. \mathrm{KOH}$ ( $40 \mathrm{mg}, 0.1$ eq.) \\ and vinyl acetate $(65 \mathrm{~mL}, 100 \mathrm{eq}$.) was stirred at RT
} for $3 \mathrm{~h}$. The reaction mixture was diluted with $\mathrm{Et}_{2} \mathrm{O}(100 \mathrm{~mL})$ and filtered through Celite. The reaction solution was concentrated in vacuo and the residue was purified on silica gel (95:5 hexanes/EtOAc) to give a clear liquid (692 mg, 56\%): $2.74\left(\mathrm{~m}, 2 \mathrm{H}, \mathrm{C}(\mathrm{O}) \mathrm{CH}_{2}\right), 3.01(\mathrm{t}, J=7.5$, $\left.2 \mathrm{H}, \mathrm{CH}_{2} \mathrm{Ph}\right), 4.60\left(\mathrm{dd}, J=6.3,1.8,1 \mathrm{H}, H_{2} \mathrm{C}=\mathrm{C}\right), 4.90\left(\mathrm{dd}, J=14.1,1.8,1 \mathrm{H}, H_{2} \mathrm{C}=\mathrm{C}\right), 7.22-7.34$ $(\mathrm{m}, 6 \mathrm{H}) ;{ }^{13} \mathrm{C}$ NMR $\delta 30.7\left(\mathrm{CH}_{2} \mathrm{Ph}\right), 35.6\left(\mathrm{C}(\mathrm{O}) \mathrm{CH}_{2}\right), 97.9\left(\mathrm{H}_{2} \mathrm{C}=\right), 126.5,128.4,128.7,140.2$ (phenyl), $141.2(=\mathrm{CH}), 170.1(\mathrm{C}=\mathrm{O})$.

Large-scale Fermentation and Purification of Subtilisin BPN' and Subtilisin E. Protease deficient $B$. subtilis DB104 was transformed with vector pBE3, as previously described. ${ }^{2,12}$ Transformed cells were grown in $2 \mathrm{XSG}^{12}$ media $(500 \mathrm{~mL})$ with shaking for $10 \mathrm{~h}$ at $37^{\circ} \mathrm{C}$. This culture was used to inoculate $14 \mathrm{~L}$ of $2 \mathrm{XSG}$ media in a $20 \mathrm{~L}$ fermentor. After 42 hours $\left(\mathrm{OD}_{600}=9.1\right)$, the culture was cooled and centrifuged. The cell pellet was discarded and the supernatant was concentrated using a $0.45 \mu \mathrm{m}$ cartridge (8,000 MW cutoff) equipped with a $1.2 \mu \mathrm{m}$ prefilter. The concentrate was brought to $70 \%$ saturation with $\mathrm{NH}_{4} \mathrm{SO}_{4}(472 \mathrm{~g} / \mathrm{L})$, stirred overnight and then centrifuged at $8,000 \mathrm{rpm}$ for $1.5 \mathrm{~h}$. The precipitate was resuspended and dialyzed for $48 \mathrm{~h}$ against $8 \mathrm{~L}$ of $10 \mathrm{mM}$ HEPES ( $\mathrm{pH} 7.5,1 \mathrm{mM} \mathrm{CaCl}_{2}$ ). The sample was passed through a bed of DE-52 anion exchange cellulose with vacuum. The clarified solution was then concentrated by passage through an 8,000 MW ultra filtration cartridge. The retentate was brought to $1.8 \mathrm{M} \mathrm{NH}_{4} \mathrm{SO}_{4}$, centrifuged and the supernatant was passed through a $0.45 \mu \mathrm{m}$ filter to give crude subtilisin.

Subtilisin E or BPN' was purified on a BioCad purification system (Applied Biosystems, Foster, USA) using a Poros 20HP2 (10 x 100) column. ${ }^{13}$ The sample was loaded and washed with $1.8 \mathrm{M} \mathrm{NH}_{4} \mathrm{SO}_{4}$ in $20 \mathrm{mM}$ HEPES (pH 7.0) and then eluted with a linear gradient of $1.8 \mathrm{M}$ to $0 \mathrm{M} \mathrm{NH}_{4} \mathrm{SO}_{3}$ in $20 \mathrm{mM}$ HEPES ( $\mathrm{pH}$ 7.0). The enzyme activity was monitored by adding $90 \mu \mathrm{L}$ of assay solution [0.2 mM succinyl-AAPF- $p$-nitroanilide (suc-AAPF- $p$ NA) / $100 \mathrm{mM}$ Tris $\mathrm{pH}$ $8.0 / 10 \mathrm{mM} \mathrm{CaCl}_{2}$ ] to $10 \mu \mathrm{L}$ of eluent and following the reaction at $410 \mathrm{~nm}$ at $37^{\circ} \mathrm{C}$ for $15 \mathrm{~min}$. The specific activity toward suc-AAPF- $p$ NA was ca. $20 \mathrm{U} / \mathrm{mg}$ (lit. ${ }^{2} 17.2 \mathrm{U} / \mathrm{mg}$ ). Fractions containing protein were concentrated by passage through an $8,000 \mathrm{MW}$ cutoff ultrafiltration cartridge and diafiltered in $10 \mathrm{mM}$ HEPES $\left(\mathrm{pH} 7.5,1 \mathrm{mM} \mathrm{CaCl}_{2}\right)$. The final retentate was frozen and lyophilized to give ca. $300 \mathrm{mg}$ of subtilisin. 


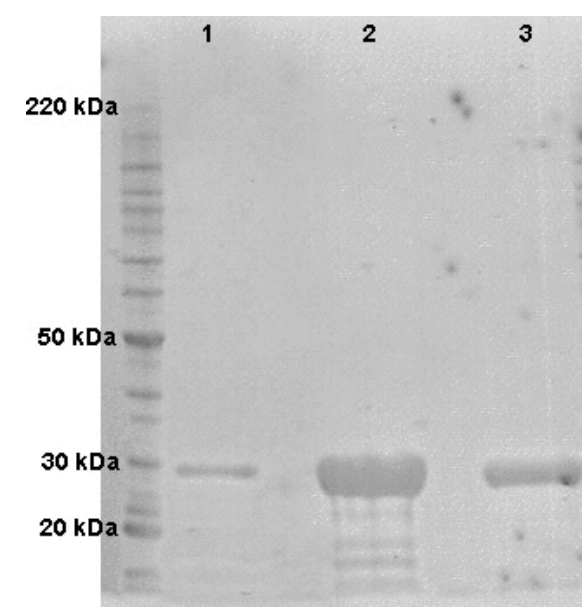

Figure S1. SDS-polyacrylamide gel electrophoresis of subtilisin $\mathrm{BPN}^{\prime}$ and $\mathrm{E}$ from $B$. subtilis DB104 carrying pBE3 and subtilisin Carlsberg (Sigma-Aldrich). The gel was stained by Coomassie Blue. To prevent autolysis, subtilisin was treated with PMSF (phenylmethylsulfonylfluoride) before denaturation. Purified PMSF-inhibited subtilisin BPN' is shown in lane 1. PMSF-inhibited subtilisin Carlsberg is shown in lane 2. Purified PMSFinhibited subtilisin $\mathrm{E}$ is shown in lane 3.

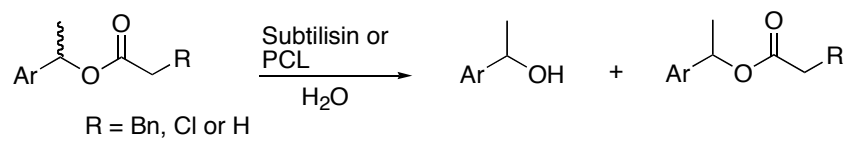

\section{Hydrolysis of esters 1a-13a with subtilisin BPN', subtilisin Carlsberg, subtilisin E or PCL. Subtilisin BPN',} subtilisin Carlsberg, subtilisin E or PCL (solution in $50 \mathrm{mM} \mathrm{BES} \mathrm{buffer}\left(\mathrm{pH} 7.2,395 \mu \mathrm{L}\right.$ ), $\mathrm{CaCl}_{2}$ $(1 \mathrm{M}, 5 \mu \mathrm{L}), 100 \mathrm{mM}$ BES buffer $(\mathrm{pH} 7.2,50 \mu \mathrm{L})$ and substrate $\left(100 \mathrm{mM}\right.$ in $\left.\mathrm{CH}_{3} \mathrm{CN}, 50 \mu \mathrm{L}\right)$ were mixed in a $1.5 \mathrm{~mL}$ polypropylene conical tube or $1.5 \mathrm{~mL}$ glass vial. The reaction was shaken at $30{ }^{\circ} \mathrm{C}$ for $48 \mathrm{~h}$ with 1a, 3a, 4a, 6a, 7a, 8a, 9a, 10a, 11a, 11c, 12a and 13a, $2 \mathrm{~h}$ with 2a and $\mathbf{5 a}$ and $3 \mathrm{~h}$ with $\mathbf{1 0 b}$ and $\mathbf{1 2 b}$. The reaction was terminated with the addition of $\mathrm{CH}_{2} \mathrm{Cl}_{2}(500$ $\mu \mathrm{L})$. The phases were separated by centrifugation, and the organic layers were collected. The aqueous phase was extracted with $\mathrm{CH}_{2} \mathrm{Cl}_{2}(2 \times 500 \mu \mathrm{L})$ and the combined organics were evaporated under a stream of air. The residue was diluted with EtOAc or EtOH $(150 \mu \mathrm{L})$ and analyzed by GC or HPLC. The enantiomers were separated using the conditions described above.

Immobilization of subtilisin BPN', subtilisin Carlsberg, subtilisin E and PCL. ${ }^{11}$ Subtilisin BPN' $(54.5 \mathrm{mg})$, subtilisin Carlsberg (52.5 mg) and subtilisin E (52.5 mg) or PCL (57.5 mg) was dissolved in $50 \mathrm{mM}$ phosphate buffer $(\mathrm{pH} 7.5,30 \mathrm{~mL})$ and $\mathrm{KCl}(10.78 \mathrm{~g})$ was added with swirling. The solution was frozen in liquid $\mathrm{N}_{2}$ and lyophilized for $48 \mathrm{~h}$.

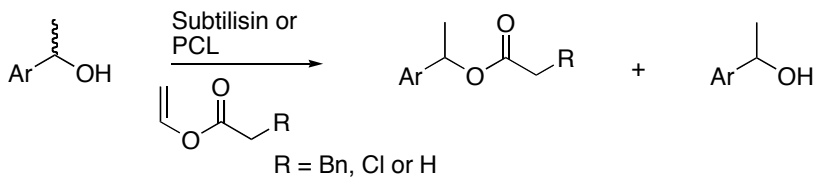
Transesterification of Secondary Alcohols 1-12 with Immobilized subtilisin BPN', subtilisin Carlsberg, subtilisin E or PCL. KCl-subtilisin or KCl-PCL powder (ca. $500 \mathrm{mg}$, ca. $2.5 \mathrm{mg}$ protein), dihydrocinnamic vinyl ester (18 mg, $100 \mu \mathrm{mol})$, chloroacetic vinyl ester $(12 \mathrm{mg}, 100 \mu \mathrm{mol})$ or vinyl acetate $(9 \mathrm{mg}, 100 \mu \mathrm{mol})$, secondary alcohol $(500 \mu \mathrm{mol})$ and 4 $\AA ̊$ molecular sieves $(100 \mathrm{mg})$ were added to a glass vial. Anhydrous dioxane $(2 \mathrm{~mL})$ was added 
and the mixture was stirred at $30{ }^{\circ} \mathrm{C}$ for at least $48 \mathrm{~h}$. A small amount of the solution was removed, filtered through a $0.45 \mu \mathrm{m}$ nylon filter and analyzed by GC or HPLC. The enantiomers were separated using the conditions described above.

Modeling of Tetrahedral Intermediates Bound to Subtilisin E. All modeling was performed using Insight II 2000.1 / Discover (Accelrys, San Diego, USA) on a SGI Octane UNIX workstation using the AMBER force field. ${ }^{14}$ We used a nonbonded cutoff distance of $8 \AA$, a distance-dependent dielectric of 1.0 and scaled the 1-4 van der Waals interactions by $50 \%$. Protein structures in Figure S2 were created using PyMOL (Delano Scientific, San Carlos, CA, USA). The X-ray crystal structure of subtilisin E (entry 1 SCJ $)^{15}$ from the Protein Data Bank is a Ser221Cys subtilisin E-propeptide complex. Using the Builder module of Insight II, we replaced the Cys221 with a serine and removed the propeptide region. The hydrogen atoms were added to correspond to $\mathrm{pH}$ 7.0. Histidines were uncharged, aspartates and glutamates were negatively charged, and arginines and lysines were positively charged. The catalytic histidine (His64) was protonated. The positions of the water hydrogens and then the enzyme hydrogens were optimized using a consecutive series of short ( $1 \mathrm{ps})$ molecular dynamic runs and energy minimizations. ${ }^{16}$ This optimization was repeated until there was $<2 \mathrm{kcal} / \mathrm{mol}$ in the energy of the minimized structures. Thereafter, an iterative series of geometry optimizations were performed on the water hydrogens, enzyme hydrogens and full water molecules. Finally, the whole system was geometry optimized.

The tetrahedral intermediates were built manually and covalently linked to Ser221. Nonstandard partial charges were calculated using a formal charge of -1 for the substrate oxyanion. Energy minimization proceeded in three stages. First, minimization of substrate with only the protein constrained $\left(25 \mathrm{kcal} \mathrm{mol}^{-1} \AA^{-2}\right)$; second, minimization with only the protein backbone constrained $\left(25 \mathrm{kcal} \mathrm{mol}^{-1} \AA^{-2}\right)$ and for the final stage the minimization was continued without constraints until the rms value was less than $0.0005 \mathrm{kcal} \mathrm{mol}^{-1} \AA^{-1}$. A catalytically productive complex required all five hydrogen bonds within the catalytic machinery. We set generous limits for a hydrogen bond: a donor to acceptor atom distance of less than $3.1 \AA$ with a nearly linear arrangement $\left(>120^{\circ}\right.$ angle) of donor atom, hydrogen, and acceptor atom. Structures lacking any of the five catalytically relevant hydrogen bonds or encountering severe steric clash with enzyme were deemed nonproductive.

Molecular Modeling Details for $(R)$-1a and $(S)$-1a Tetrahedral Intermediates bound to Subtilisin E. Modeling 1a with subtilisin E gave one productive conformation for each enantiomer. The two other plausible conformations encountered severe steric clash with the protein. Similar to previous modeling studies with subtilisin Carlsberg, ${ }^{17}$ the productive conformation of the $(S)$-1a binds with the methyl group in the leaving-group $S_{1}{ }^{\prime}$ pocket and the productive conformation of the $(R)-\mathbf{1 a}$ has the phenyl group in this pocket.

Productive Conformer of $(R)$-1a (Figure S2). $(R)$-1a fit well in the active site, but $\mathrm{S}_{1}{ }^{\prime}$ pocket residues bumped the phenyl group $\left(\mathrm{C}_{\text {ortho }}-\mathrm{C}_{\varepsilon}\right.$ (Met222) distance $=3.77 \AA, \mathrm{C}_{\text {meta }}-\mathrm{C}_{\gamma}$ (Tyr217) distance $=3.68 \AA, \mathrm{C}_{\text {para }}-\mathrm{C}_{\beta}(\mathrm{Tyr} 217)$ distance $=3.72 \AA$ and $\mathrm{C}_{\text {ortho }}-\mathrm{C}_{\alpha}($ Gly219) distance $=3.72 \AA) .{ }^{18}$ The increased steric interaction between $\mathrm{S}_{1}{ }^{\prime}$ residues and the phenyl group may hinder binding of $(R)-\mathbf{1 a}$ and account for the enantiopreference of subtilisin $\mathrm{E}$ for $(S)-\mathbf{1 a}$ in organic solvent. However, this tight fit also suggests a favorable hydrophobic interaction tendency of nonpolar compounds to transfer from an aqueous phase to an organic phase - 
between the phenyl group and $\mathrm{S}_{1}{ }^{\text {}}$ pocket residues. ${ }^{19}$ This favorable hydrophobic interaction may improve binding of the phenyl group and account for the enantiopreference with secondary alcohols in water. In nonpolar media, this favorable hydrophobic effect is expected to be negligible, thus the increased steric hindrance experienced by the phenyl group versus the methyl group in the $S_{1}{ }^{\prime}$ pocket would favor reaction of $(S)-\mathbf{1 a}$.

Non-productive Conformer of $(R)$-1a (not shown). The methyl group of nonproductive $(R)$-1a bumped catalytic His $64\left(\mathrm{CH}_{3}-\mathrm{C}_{\delta 2}\right.$ distance $=3.58 \AA$ and the phenyl group encountered steric clash with the oxyanion hole Asn155 $\left(\mathrm{C}_{\mathrm{ipso}}-\mathrm{N}_{\delta 2}\right.$ distance $=3.41$ and $\mathrm{C}_{\text {ortho }}-\mathrm{N}_{\delta 2}$ distance $=3.29 \AA) .{ }^{18}$ The methyl group was forced out of the $S_{1}{ }^{\prime}$ leaving-group pocket because of this steric clash with active site residues.

Productive Conformer of $(S)$-1a (Figure S2). $(S)$-1a fit well in the active site and $S_{1}{ }^{\prime}$ residues did not hinder the methyl group.

Non-productive Conformer of $(S)$-1a (not shown). The phenyl group of non-productive (S)-1a encountered steric clash with catalytic His $64\left(\mathrm{C}_{\mathrm{ipso}}-\mathrm{C}_{\delta 2}\right.$ distance $=3.53 \AA, \mathrm{C}_{\text {ortho }}-\mathrm{N}_{\varepsilon 2}$ distance $=3.53 \AA, \mathrm{C}_{\text {ortho }}-\mathrm{C}_{\delta 2}$ distance $=3.44 \AA$ and $\mathrm{C}_{\text {meta }}-\mathrm{C}_{\delta 2}$ distance $=3.52 \AA$ ) and the methyl group bumped the oxyanion hole Asn $155\left(\mathrm{C}_{\text {methyl }}-\mathrm{N}_{\delta 2}\right.$ distance $\left.=3.68 \AA\right) .{ }^{18}$ The phenyl group was forced out of the $S_{1}{ }^{\prime}$ leaving-group pocket because of this steric clash with active site residues.
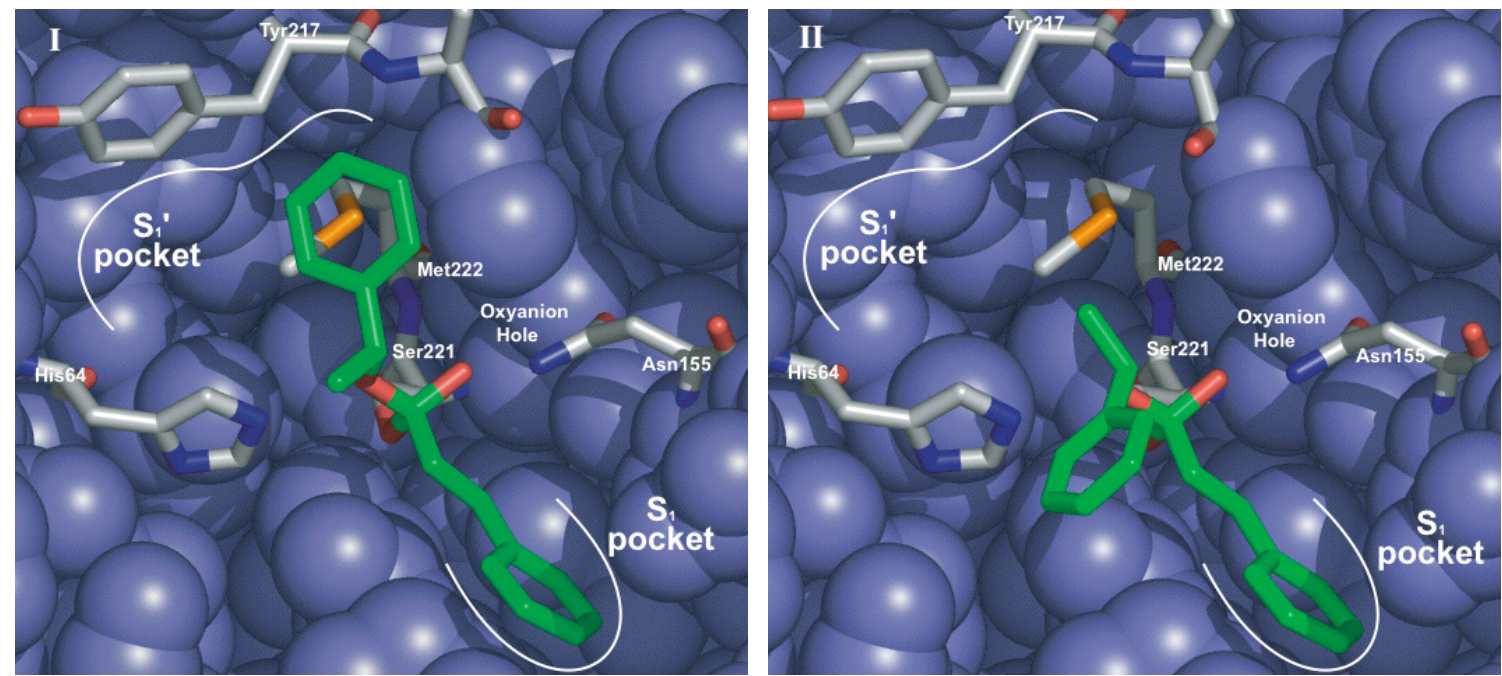

Figure S2. Catalytically competent orientation of the tetrahedral intermediates for the subtilisin E catalyzed hydrolysis of $(R)-\mathbf{1 a}(\mathrm{I})$ and $(S)$-1a (II) as identified by molecular modeling. The important active site and substrate atoms are shown as sticks. The atoms are colored as follows: green (substrate carbon), gray (enzyme carbon), red (oxygen), blue (nitrogen) and orange (sulfur). Surrounding atoms (space fill) of subtilisin are shown in the color blue. For clarity, all hydrogen atoms and water molecules are hidden. The binding modes of I and II maintain all catalytically essential hydrogen bonds and the benzyl moiety of the dihydrocinnamoyl group was bound in the $S_{1}$ pocket, as expected based on its similarity to phenylalanine. On the basis of previous work with secondary alcohols, the phenyl group (Large) or methyl group (Medium) was 
bound in the leaving-group $\mathrm{S}_{1}{ }^{\prime}$ pocket. ${ }^{17}$ In the fast-reacting enantiomer, $(R)$-1a $(\mathrm{I})$, the phenyl group is bound in the hydrophobic $S_{1}{ }^{\prime}$ pocket and the methyl group is exposed to solvent water. In the slow-reacting enantiomer, $(S)$-1a (II), the methyl group is bound in the hydrophobic $\mathrm{S}_{1}{ }^{\prime}$ pocket and the phenyl group is exposed to solvent water. The non-productive conformations (not shown) encountered severe steric clash with the active site residues of the protein.

Table S1. Minimized structures for the tetrahedral intermediate for the subtilisin Ecatalyzed hydrolysis of $\mathbf{1 a}$.

\begin{tabular}{|c|c|c|c|}
\hline Conformation & $\begin{array}{l}\text { Group } \\
\text { in } \mathrm{S}_{1}{ }^{a}\end{array}$ & $\begin{array}{l}\mathrm{H} \text {-bond } \mathrm{N}_{\varepsilon 2}-\mathrm{O}_{\gamma} \\
\text { distance }(\AA)(\mathrm{N}- \\
\left.\mathrm{H}-\mathrm{O}_{\gamma} \text { angle, deg }\right)^{b}\end{array}$ & Comments \\
\hline$(R)-\mathbf{1 a}$ (Figure S2) & phenyl & $2.94(136)$ & $\begin{array}{l}\text { Steric contact with Tyr } 217 \text {, } \\
\text { Gly } 219 \text { and Met } 222 \text { in } S_{1}{ }^{\prime} \\
\text { pocket }\end{array}$ \\
\hline$(R)-\mathbf{1 a}($ not shown) & methyl $^{\mathrm{c}}$ & $2.99(134)$ & $\begin{array}{l}\text { Steric clash with catalytic } \\
\text { residues His } 64 \text { and Asn } 155\end{array}$ \\
\hline$(S)-\mathbf{1 a}$ (Figure S2) & methyl & $2.95(138)$ & Unhindered in $S_{1}{ }^{\prime}$ pocket \\
\hline$(S)-\mathbf{1 a}($ not shown) & phenyl $^{\mathrm{c}}$ & $3.11(135)$ & $\begin{array}{l}\text { Steric clash with catalytic } \\
\text { residues His } 64 \text { and Asn } 155\end{array}$ \\
\hline
\end{tabular}

Tables of Enantioselectivity. Tables S2-S4 support Table 1. They provide the enantiomer excess and enantioselectivity data for subtilisin BPN ', Carlsberg and E-catalyzed hydrolysis of esters 1a-13a, 10b and 12b and transesterification of secondary alcohols 1-12. Table S5 provides the enantiomer excess and enantioselectivity data for PCL-catalyzed hydrolysis of esters 1a-13a and 10b, 11c and 12b and transesterification of 1-12.

Table S2. Enantioselectivity of BPN'-catalyzed hydrolysis of 1a-13a and transesterification of 113 with a vinyl ester in dioxane.

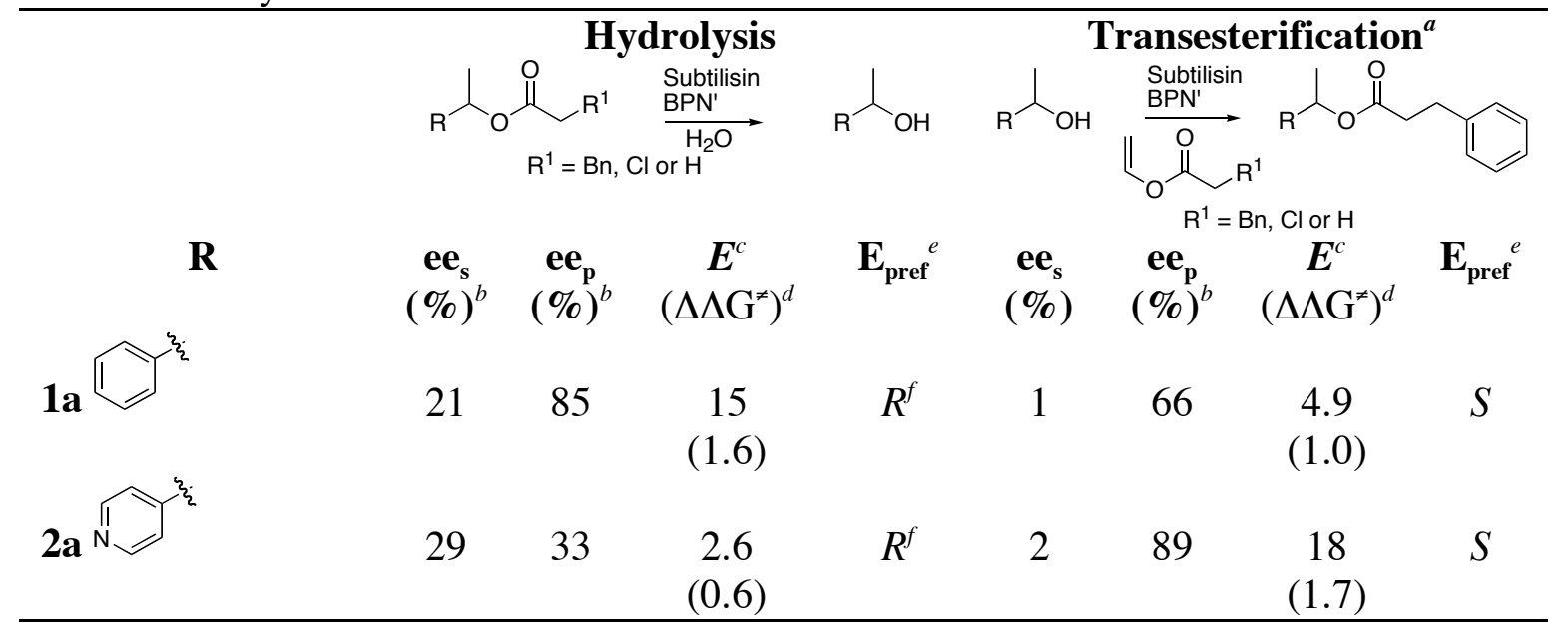




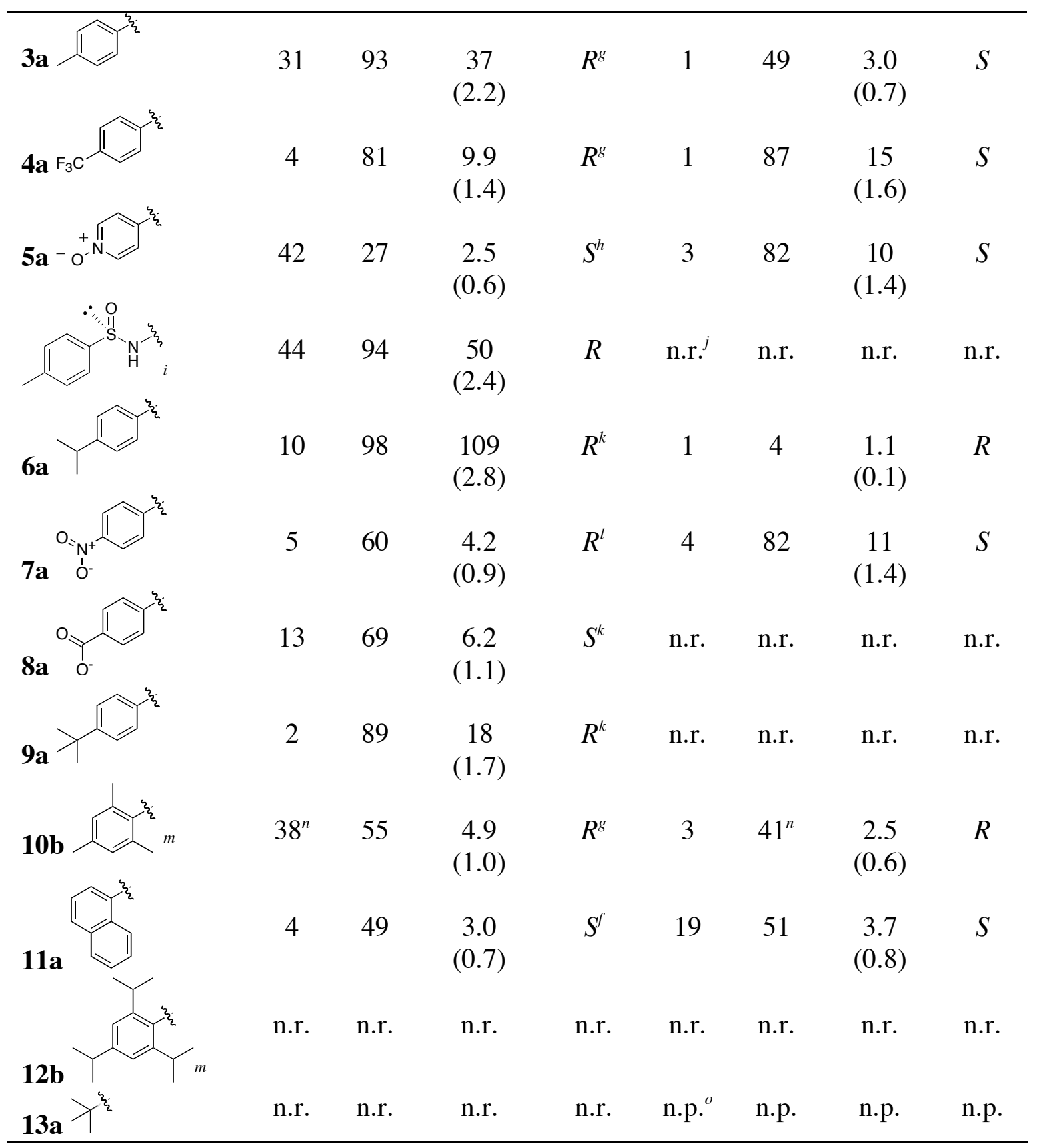

${ }^{a}$ Enzyme immobilized on KCL. ${ }^{b}$ Enantiomeric excess: enantiomeric excess of substrate and product were determined by GC analysis on $25 \mathrm{~m}$ x $0.25 \mathrm{~mm}$ Chrompack CP-Chiralsil-Dex CB column (Varian Inc., Palo Alto, CA, USA) using He as a carrier gas or HPLC analysis performed on a 4.6 x $250 \mathrm{~mm}$ Daicel Chiralcel OD or Chrialpak AD-H column (Chiral Technologies, Exton, USA) and monitored at $254 \mathrm{~nm}$. 'Enantioselectivity: the enantiomeric ratio $E$ measures the relative rate of hydrolysis of the fast enantiomer as compared to the slow enantiomer as defined by Sih (Chen, C.S.; Fujimoto, Y.; Girdaukas, G.; Sih, C.J. J. Am. Chem. Soc. 1982, 104, 72947299). ${ }^{d} \Delta \Delta \mathrm{G}^{\neq}=-\mathrm{RT} \ln \mathrm{V}_{\mathrm{A} /} / \mathrm{V}_{\mathrm{B}}$, where $\mathrm{V}_{\mathrm{A}}$ and $\mathrm{V}_{\mathrm{B}}$ are the reaction rates of enantiomers $\mathrm{A}$ and $\mathrm{B}$, respectively. $\Delta \Delta \mathrm{G}^{7}$ is the difference in activation energy of the enantiomeric substrates. This is a direct measure for the selectivity of reaction, which in turn depends on the ratio of the individual 
reaction rates of the enantiomers (see Faber, K. Biotransformations in Organic Chemistry; Springer-Verlag: Berlin, 1997; pp 17-20.). ${ }^{e}$ Enantiopreference. ${ }^{f}$ Absolute configuration determined by comparison with an authentic sample (Sigma-Aldrich). ${ }^{g}$ Absolute configuration determined by similarity in the order of elution in the GC analysis (Doucet, H.; Fernandez, E.; Layzell, T. P.; Brown, J. M. Chem. Eur. J. 1999, 5, 1320-1330.). ${ }^{h}$ Absolute configuration determined by comparison with an authentic sample prepared as described above. ${ }^{I} N$ -

Dihydrocinnamoyl-p-toluenesulfinamide. From Mugford, P. F.; Magloire, V. M.; Kazlauskas, R. J. J. Am. Chem. Soc. 2005, 127, 0000-0000. ${ }^{j}$ No reaction. ${ }^{k}$ Absolute configuration determined using the configurational correlation model for the corresponding $(R)$-MTPA derivatives (Dale, J. A.; Mosher, H. S. J. Am. Chem. Soc. 1973, 95, 512-519.). 'Absolute configuration determined by similarity in the order of elution in the GC analysis (Uray, G.; Stampfer, W.; Fabian, W. M. F. J. Chromatogr. A 2003, 992, 151-157.). ${ }^{m}$ Reaction as chloroacetate. ${ }^{n} \mathbf{1 0 b}$ could not be separated by GC or HPLC. To facilitate analysis, 10b was purified on silica gel (100\% hexanes to 90:10 hexanes/EtOAc) and hydrolyzed in $1 \mathrm{~N} \mathrm{KOH}\left(1: 1 \mathrm{EtOH} / \mathrm{H}_{2} \mathrm{O}\right)$ to $\mathbf{1 0}$ for $\mathrm{GC}$ analysis. ${ }^{o}$ Not performed.

Table S3. Enantioselectivity of subtilisin Carlsberg-catalyzed hydrolysis of 1a-13a and transesterification of 1-13 with a vinyl ester in dioxane.

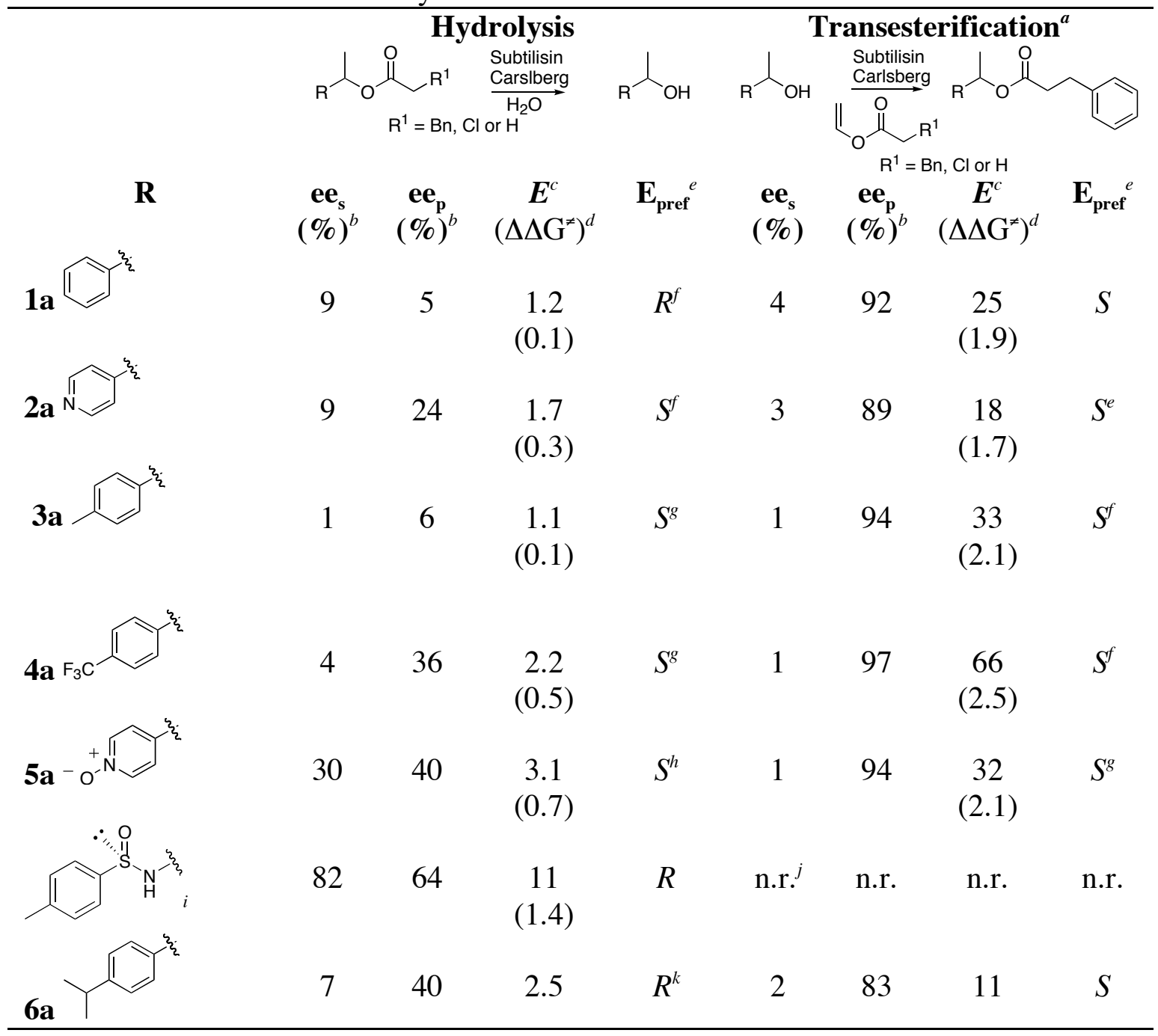




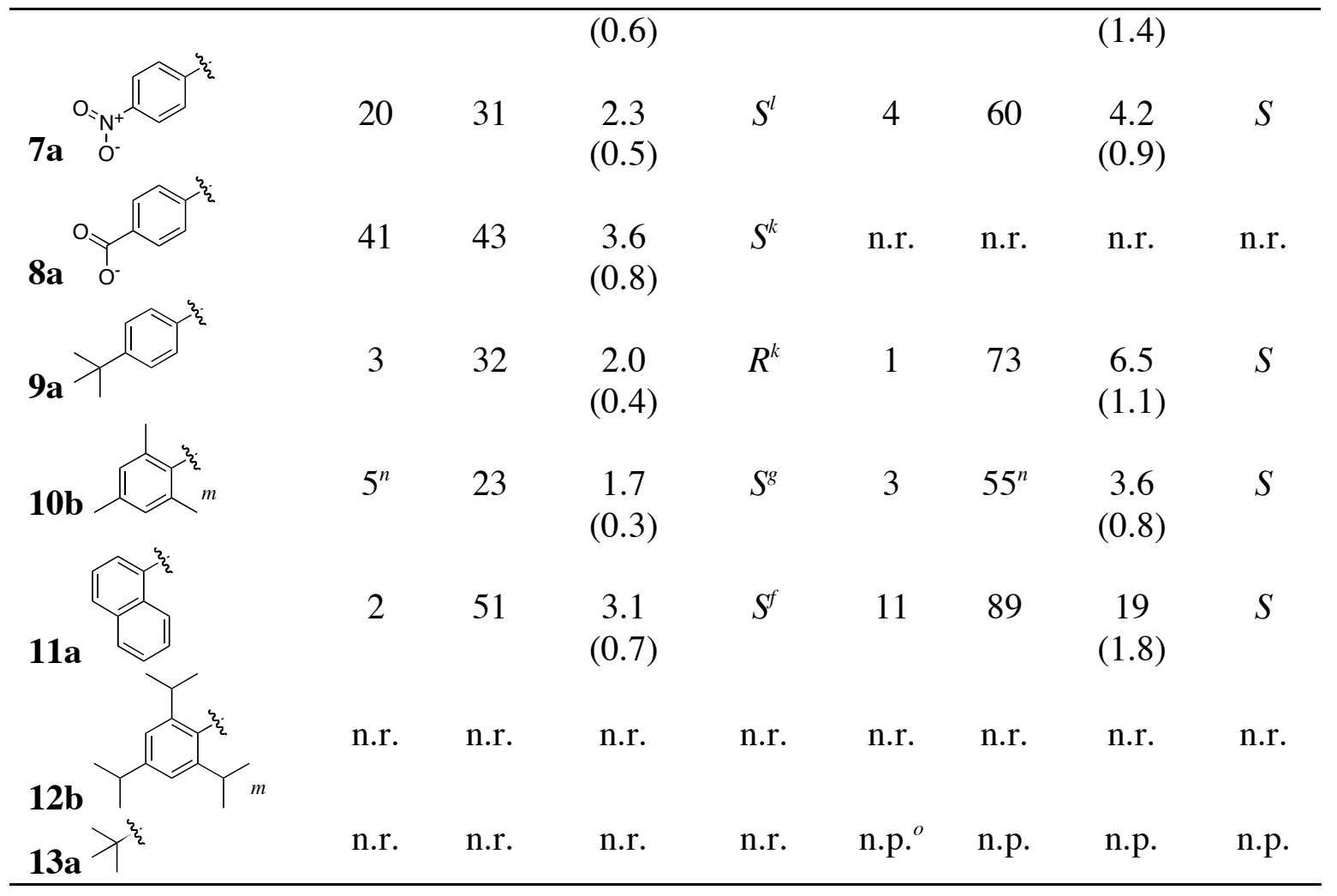

${ }^{a}$ Enzyme immobilized on KCL. ${ }^{b}$ Enantiomeric excess: enantiomeric excess of substrate and product were determined by GC analysis on $25 \mathrm{~m}$ x $0.25 \mathrm{~mm}$ Chrompack CP-Chiralsil-Dex CB column (Varian Inc., Palo Alto, CA, USA) using He as a carrier gas or HPLC analysis performed on a 4.6 x $250 \mathrm{~mm}$ Daicel Chiralcel OD or Chrialpak AD-H column (Chiral Technologies, Exton, USA) and monitored at $254 \mathrm{~nm}$. ${ }^{c}$ Enantioselectivity: the enantiomeric ratio $E$ measures the relative rate of hydrolysis of the fast enantiomer as compared to the slow enantiomer as defined by Sih (Chen, C.S.; Fujimoto, Y.; Girdaukas, G.; Sih, C.J. J. Am. Chem. Soc. 1982, 104, 72947299). ${ }^{d} \Delta \Delta \mathrm{G}^{\neq}=-\mathrm{RT} \ln \mathrm{V}_{\mathrm{A} /} / \mathrm{V}_{\mathrm{B}}$, where $\mathrm{V}_{\mathrm{A}}$ and $\mathrm{V}_{\mathrm{B}}$ are the reaction rates of enantiomers $\mathrm{A}$ and $\mathrm{B}$, respectively. $\Delta \Delta \mathrm{G}^{\neq}$is the difference in activation energy of enantiomeric substrates. This is a direct measure for the selectivity of reaction, which in turn depends on the ratio of the individual reaction rates of the enantiomers (see Faber, K. Biotransformations in Organic Chemistry; Springer-Verlag: Berlin, 1997; pp 17-20.). ${ }^{e}$ Enantiopreference. ${ }^{f}$ Absolute configuration determined by comparison with an authentic sample (Sigma-Aldrich). ${ }^{g}$ Absolute configuration determined by similarity in the order of elution in the GC analysis (Doucet, H.; Fernandez, E.; Layzell, T. P.; Brown, J. M. Chem. Eur. J. 1999, 5, 1320-1330.). ${ }^{h}$ Absolute configuration determined by comparison with an authentic sample prepared as described above. ${ }^{i} N$ Dihydrocinnamoyl-p-toluenesulfinamide. From Savile, C. K.; Magloire, V. M.; Kazlauskas, R. J. J. Am. Chem. Soc. 2005, 127, 2104-2113. ${ }^{j}$ No reaction. ${ }^{k}$ Absolute configuration determined using the configurational correlation model for the corresponding $(R)$-MTPA derivatives (Dale, J. A.; Mosher, H. S. J. Am. Chem. Soc. 1973, 95, 512-519.). 'Absolute configuration determined by similarity in the order of elution in the GC analysis (Uray, G.; Stampfer, W.; Fabian, W. M. F. J. Chromatogr. A 2003, 992, 151-157.). ${ }^{m}$ Reaction as chloroacetate. ${ }^{n} \mathbf{1 0 b}$ could not be separated by GC or HPLC. To facilitate analysis, 10b was purified on silica gel (100\% hexanes to 90:10 hex- 
anes/EtOAc) and hydrolyzed in $1 \mathrm{~N} \mathrm{KOH}\left(1: 1 \mathrm{EtOH} / \mathrm{H}_{2} \mathrm{O}\right)$ to $\mathbf{1 0}$ for $\mathrm{GC}$ analysis. ${ }^{\circ}$ Not performed.

Table S4. Enantioselectivity of subtilisin E-catalyzed hydrolysis of 1a-13a and transesterification of 1-13 with a vinyl ester in dioxane.

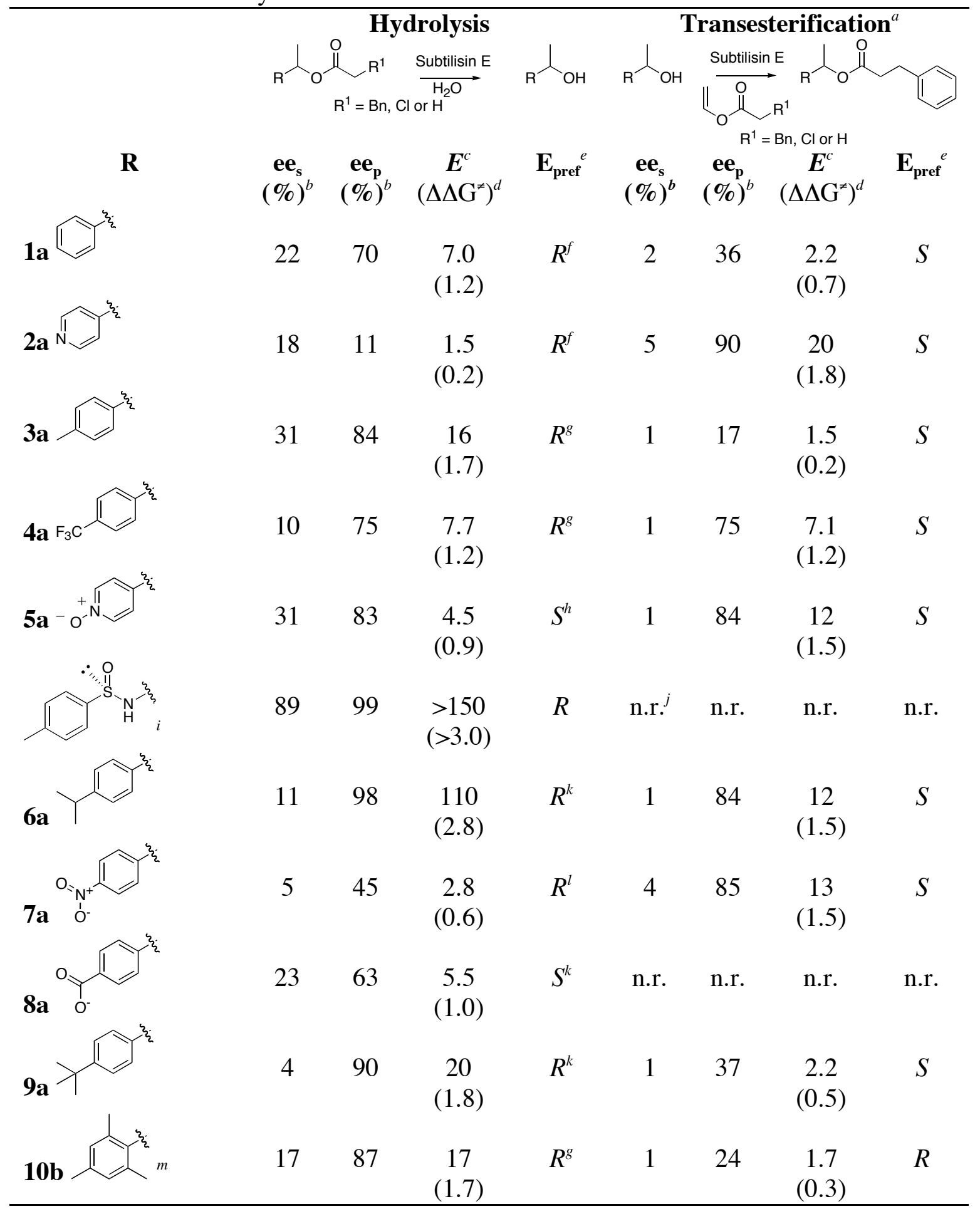




\begin{tabular}{|c|c|c|c|c|c|c|c|c|}
\hline 11a & 6 & 26 & $\begin{array}{c}1.8 \\
(0.4)\end{array}$ & $R^{f}$ & 6 & 58 & $\begin{array}{c}4.0 \\
(0.8)\end{array}$ & $S$ \\
\hline & n.r. & n.r. & n.r. & n.r. & n.r. & n.r. & n.r. & n.r. \\
\hline $13 a$ & n.r. & n.r. & n.r. & n.r. & n.p. ${ }^{o}$ & n.p. & n.p. & n.p. \\
\hline
\end{tabular}

${ }^{a}$ Enzyme immobilized on KCL. ${ }^{b}$ Enantiomeric excess: enantiomeric excess of substrate and product were determined by GC analysis on $25 \mathrm{~m}$ x $0.25 \mathrm{~mm}$ Chrompack CP-Chiralsil-Dex CB column (Varian Inc., Palo Alto, CA, USA) using He as a carrier gas or HPLC analysis performed on a 4.6 x $250 \mathrm{~mm}$ Daicel Chiralcel OD or Chrialpak AD-H column (Chiral Technologies, Exton, USA) and monitored at $254 \mathrm{~nm}$. ${ }^{c}$ Enantioselectivity: the enantiomeric ratio $E$ measures the relative rate of hydrolysis of the fast enantiomer as compared to the slow enantiomer as defined by Sih (Chen, C.S.; Fujimoto, Y.; Girdaukas, G.; Sih, C.J. J. Am. Chem. Soc. 1982, 104, 72947299). ${ }^{d} \Delta \Delta \mathrm{G}^{\neq}=-\mathrm{RT} \ln \mathrm{V}_{\mathrm{A} /} / \mathrm{V}_{\mathrm{B}}$, where $\mathrm{V}_{\mathrm{A}}$ and $\mathrm{V}_{\mathrm{B}}$ are the reaction rates of enantiomers $\mathrm{A}$ and $\mathrm{B}$, respectively. $\Delta \Delta \mathrm{G}^{\neq}$is the difference in activation energy of enantiomeric substrates. This is a direct measure for the selectivity of reaction, which in turn depends on the ratio of the individual reaction rates of the enantiomers (see Faber, K. Biotransformations in Organic Chemistry; Springer-Verlag: Berlin, 1997; pp 17-20.). ${ }^{e}$ Enantiopreference. ${ }^{f}$ Absolute configuration determined by comparison with an authentic sample (Sigma-Aldrich). ${ }^{g}$ Absolute configuration determined by similarity in the order of elution in the GC analysis (Doucet, H.; Fernandez, E.; Layzell, T. P.; Brown, J. M. Chem. Eur. J. 1999, 5, 1320-1330.). ${ }^{h}$ Absolute configuration determined by comparison with an authentic sample prepared as described above. ${ }^{I} N$ Dihydrocinnamoyl-p-toluenesulfinamide. From Savile, C. K.; Magloire, V. M.; Kazlauskas, R. J. J. Am. Chem. Soc. 2005, 127, 2104-2113. ${ }^{j}$ No reaction. ${ }^{k}$ Absolute configuration determined using the configurational correlation model for the corresponding $(R)$-MTPA derivatives (Dale, J. A.; Mosher, H. S. J. Am. Chem. Soc. 1973, 95, 512-519.). ' Absolute configuration determined by similarity in the order of elution in the GC analysis (Uray, G.; Stampfer, W.; Fabian, W. M. F. $J$. Chromatogr. A 2003, 992, 151-157.). ${ }^{m}$ Reaction as chloroacetate. ${ }^{n} \mathbf{1 0 b}$ could not be separated by GC or HPLC. To facilitate analysis, 10b was purified on silica gel (100\% hexanes to 90:10 hexanes/EtOAc) and hydrolyzed in $1 \mathrm{~N} \mathrm{KOH}\left(1: 1 \mathrm{EtOH} / \mathrm{H}_{2} \mathrm{O}\right)$ to $\mathbf{1 0}$ for $\mathrm{GC}$ analysis. ${ }^{o}$ Not performed.

Table S5. Enantioselectivity of PCL-catalyzed hydrolysis of 1a-13a or transesterification of 1-13 with a vinyl ester in dioxane.

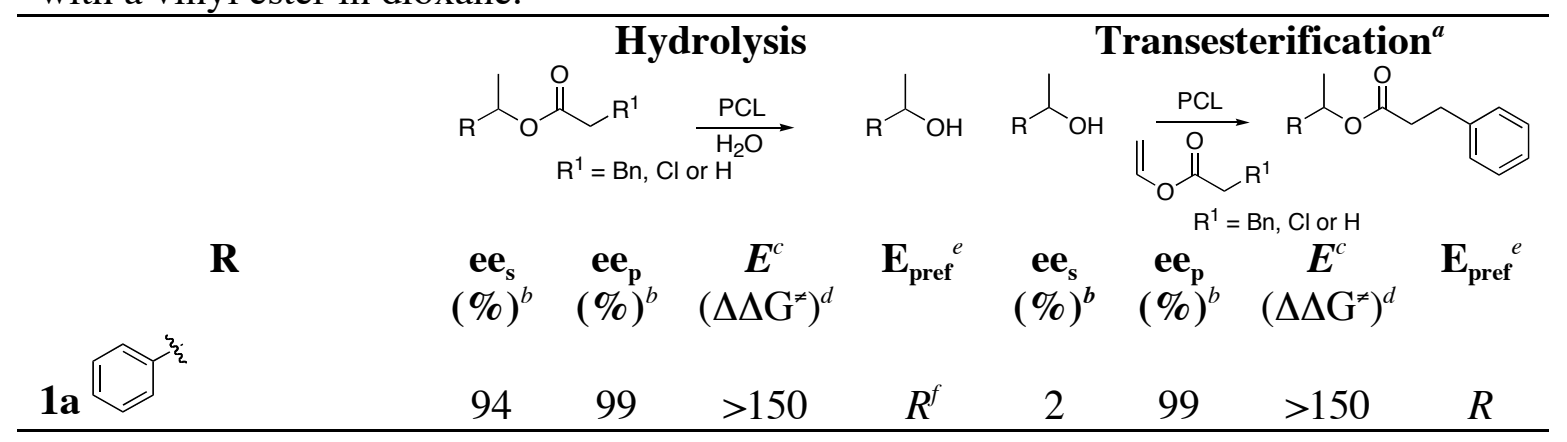




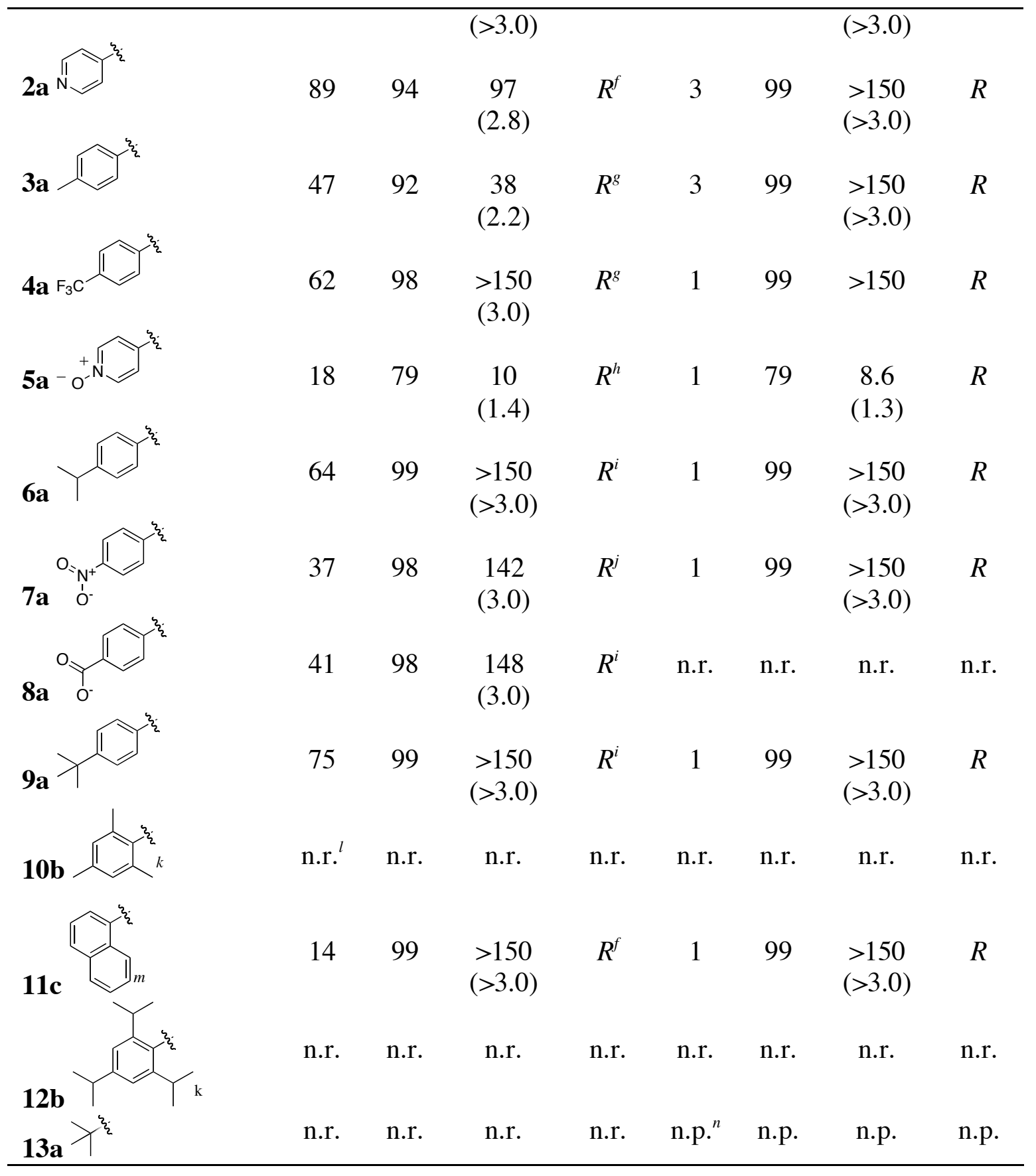

${ }^{a}$ Enzyme immobilized on KCL. ${ }^{b}$ Enantiomeric excess: enantiomeric excess of substrate and product were determined by GC analysis on $25 \mathrm{~m}$ x $0.25 \mathrm{~mm}$ Chrompack CP-Chiralsil-Dex CB column (Varian Inc., Palo Alto, CA, USA) using He as a carrier gas or HPLC analysis performed on a 4.6 x $250 \mathrm{~mm}$ Daicel Chiralcel OD or Chrialpak AD-H column (Chiral Technologies, Exton, USA) and monitored at $254 \mathrm{~nm}$. ${ }^{c}$ Enantioselectivity: the enantiomeric ratio $E$ measures the relative rate of hydrolysis of the fast enantiomer as compared to the slow enantiomer as defined by Sih (Chen, C.S.; Fujimoto, Y.; Girdaukas, G.; Sih, C.J. J. Am. Chem. Soc. 1982, 104, 72947299). ${ }^{d} \Delta \Delta \mathrm{G}^{\neq}=-\mathrm{RT} \ln \mathrm{V}_{\mathrm{A}} / \mathrm{V}_{\mathrm{B}}$, where $\mathrm{V}_{\mathrm{A}}$ and $\mathrm{V}_{\mathrm{B}}$ are the reaction rates of enantiomers $\mathrm{A}$ and $\mathrm{B}$, respectively. $\Delta \Delta \mathrm{G}^{\neq}$is the difference in activation energy of enantiomeric substrates. This is a di- 
rect measure for the selectivity of reaction, which in turn depends on the ratio of the individual reaction rates of the enantiomers (see Faber, K. Biotransformations in Organic Chemistry; Springer-Verlag: Berlin, 1997; pp 17-20.). ${ }^{e}$ Enantiopreference. ${ }^{f}$ Absolute configuration determined by comparison with an authentic sample (Sigma-Aldrich). ${ }^{g}$ Absolute configuration determined by similarity in the order of elution in the GC analysis (Doucet, H.; Fernandez, E.; Layzell, T. P.; Brown, J. M. Chem. Eur. J. 1999, 5, 1320-1330.). ${ }^{h}$ Absolute configuration determined by comparison with an authentic sample prepared as described above. ${ }^{i}$ Absolute configuration determined using the configurational correlation model for the corresponding $(R)$-MTPA derivatives (Dale, J. A.; Mosher, H. S. J. Am. Chem. Soc. 1973, 95, 512-519.). ${ }^{j}$ Absolute configuration determined by similarity in the order of elution in the GC analysis (Uray, G.; Stampfer, W.; Fabian, W. M. F. J. Chromatogr. A 2003, 992, 151-157.). ${ }^{k}$ Reaction as chloroacetate. ${ }^{l}$ No reaction. ${ }^{m}$ Reaction as acetate. ${ }^{o}$ Not performed.

\section{References}

1. Lipase from Burkholderia cepacia was formerly known as lipase from Pseudomonas cepacia.

2. Zhao, H.; Arnold, F. H. Proc. Natl. Acad. Sci. USA 1997, 94, 7997-8000.

3. Kawamura, F.; Doi, R. H. J. Bacteriol. 1984, 160, 442-444.

4. Lin, Y. Y.; Palmer, D. N.; Jones, J. B. Can. J. Chem. 1974, 52, 469-476.

5. Craig, J. C.; Purushothaman, K. K. J. Org. Chem. 1970, 35, 1721-1722.

6. Taylor, R. J. Chem. Soc., Perkin Trans. 2 1975, 277-281.

7. Doucet, H.; Fernandez, E.; Layzell, T. P.; Brown, J. M. Chem. Eur. J. 1999, 5, 1320-1330.

8. Uray, G.; Stampfer, W.; Fabian, W. M. F. J. Chromatogr. A 2003, 992, 151-157.

9. Delair, P.; Kanazawa, A. M.; de Azevedo, M. B. M.; Greene, A. E. Tetrahedron: Asymmetry 1996, 7, 2707-2710.

10. Dale, J. A.; Mosher, H. S. J. Am. Chem. Soc. 1973, 95, 512-519.

11. Lloyd, R. C.; Dickman, M.; Jones, J. B. Tetrahedron: Asymmetry 1998, 9, 551-561.

12. Harwood, C. R.; Cutting, S. M. Molecular Biological Methods for Bacillus, John Wiley \&

Sons: Chichester, U.K., 1990; pp 33-35 and 391-402.

13. Cho, S.-J.; OH, S.-H.; Pridmore, R. D.; Juillerat, M. A.; Lee, C.-H. J. Agric. Food Chem. 2003, 51, 7664-7670.

14. (a) Weiner, S. J.; Kollman, P. A.; Case, D. A.; Singh, U. C.; Ghio, C.; Alagona, G.; Profeta, S.; Weiner, P. J. Am. Chem. Soc. 1984, 106, 765-784. (b) Weiner, S. J.; Kollman, P. A.; Nguyen, D. T.; Case, D. A. J. Comp. Chem. 1986, 7, 230-252.

15. Jain, S. C.; Shinde, U.; Li, Y.; Inouye, M.; Berman, H. M. J. Mol. Biol. 1998, 284, 137-144. 16. Raza, S.; Fransson, L.; Hult, K. Protein Sci. 2001, 10, 329-338.

17. Fitzpatrick, P. A.; Ringe, D.; Klibanov, A. M. Biotechnol. Bioeng. 1992, 40, 735-742.

18. The van der Waals distance for $\mathrm{CH}-\mathrm{C}=3.99 \AA, \mathrm{CH}-\mathrm{N}=3.84 \AA$ and $\mathrm{O}-\mathrm{HC}=3.81 \AA$. These were estimated from the van der Waals radii of carbon $(1.70 \AA)$, nitrogen $(1.55 \AA)$ or oxygen $(1.52 \AA)$ and hydrogen $(1.20 \AA)$ and the $\mathrm{C}-\mathrm{H}$ bond length $(1.09 \AA)$ or O-H bond length $(0.96 \AA)$ from Bondi, A. J. Phys. Chem. 1964, 68, 441-451.

19. The energy of a hydrophobic interaction is from the regaining of entropy by water after it is removed from a hydrophobic group and can be estimated using the incremental Gibbs free energy of transfer. ${ }^{20,21}$ The incremental Gibbs free energy of transfer of the phenyl group from $n$ octanol to water was estimated to be ca. $+2.9 \mathrm{kcal} / \mathrm{mol}$, using $\Delta \mathrm{G}_{\text {trans }}=2.303 R T \pi$, where $\pi$ is the hydrophobicity constant of the phenyl group $(\pi=2.1)^{22}$ relative to hydrogen, ${ }^{20}$ whereas the 
methyl group was ca. $+1.5 \mathrm{kcal} / \mathrm{mol}$. Thus, the energy difference between the two groups is ca. $1.4 \mathrm{kcal} / \mathrm{mol}$ in favor of the phenyl group when in an aqueous medium.

20. Fersht, A. Structure and Mechanism in Protein Science; W.H. Freeman and Company: New York, 1998; pp 324-348.

21. Leo, A.; Hansch, C.; Elkins, D. Chem. Rev. 1971, 71, 525-616.

22. Hydrophobicity partition coefficients $(\log P)$ values were calculated using a computer program, $C \log P$ (BioByte, Claremont, USA). 